\title{
Generalized Fokker-Planck theory for electron and photon transport in biological tissues: application to radiotherapy
}

\author{
Edgar Olbrant* and Martin Frank \\ Department of Mathematics, Center for Computational Engineering Science, RWTH Aachen \\ University, Schinkelstrasse 2, D-52062 Aachen, Germany
}

(Received 9 December 2009; final version received 21 April 2010)

\begin{abstract}
In this paper, we study a deterministic method for particle transport in biological tissues. The method is specifically developed for dose calculations in cancer therapy and for radiological imaging. Generalized Fokker-Planck (GFP) theory [Leakeas and Larsen, Nucl. Sci. Eng. 137 (2001), pp. 236-250] has been developed to improve the Fokker-Planck (FP) equation in cases where scattering is forward-peaked and where there is a sufficient amount of large-angle scattering. We compare grid-based numerical solutions to FP and GFP in realistic medical applications. First, electron dose calculations in heterogeneous parts of the human body are performed. Therefore, accurate electron scattering cross sections are included and their incorporation into our model is extensively described. Second, we solve GFP approximations of the radiative transport equation to investigate reflectance and transmittance of light in biological tissues. All results are compared with either Monte Carlo or discrete-ordinates transport solutions.
\end{abstract}

Keywords: generalized Fokker-Planck; deterministic method; radiotherapy; particle transport; Boltzmann equation; Monte Carlo

AMS Subject Classification: 35Q20; 35Q84; 65C05; 62P10; 78A35; 85A25

\section{Introduction}

The numerical solution of the Boltzmann transport equation (BTE) $[7,20]$ remains a difficult and important challenge in electron and photon transport. To contribute to this field of research, we study selected approximations of the transport equation for electrons and photons, and present numerical results in different geometries.

Nowadays, cancer patients often undergo therapies with high energy ionizing radiation. In external radiotherapy, photon beams dominate in clinical use - less patients receive electron therapy. Treatments are also performed by using heavy-charged particles such as ions or protons. These have higher costs for their particle accelerators, but gain more and more importance due to first high-intensity laser systems for protons [47].

To aid the recovery of patients, it is important to deposit a sufficient amount of energy in the tumour. Simultaneously, the ambient healthy tissue should not be damaged. Therefore, the success of such radiation treatments strongly depends on the correct dose distribution. It is recommended that uncertainties in dose distributions should be less than $2 \%$ to get an overall desired accuracy of $3 \%$ in the delivered dose to a volume [43].

\footnotetext{
*Corresponding author. Email: olbrant@ mathcces.rwth-aachen.de
} 
Additionally, thresholds have been developed to compare dose results computed by different algorithms. A suggested tolerance in homogeneous geometries is the $2 \%$ (relative pointwise difference) or $2 \mathrm{~mm}$ (absolute distance to agreement) criterion. However, in heterogeneities this limit increases to $3 \%$ or $3 \mathrm{~mm}$ [53].

Up to now, many clinical dose calculation algorithms rely on pencil beam models. Originally developed for cosmic ray showers, Fermi ([16], cited by [45]) and Eyges [15] introduced a small-angle scattering theory which was later applied to electrons. This theory was used by Hogstrom et al. to propose the pencil-beam model. Their algorithm includes experimental data, taken from dose measurements in a water phantom, to compute the central-axis dose [26]. Although it was the first clinically applicable model, its accuracy deep in the irradiated material or in heterogeneities is poor. This is basically due to the small-angle approximation in Fermi-Eyges theory. It is true that single electron collisions show small deviations. However, after multiple scattering events, they accumulate to big angle changes in large penetration depths. Besides, crude approximations such as small deviations of particles throughout their whole path through the tissue [37], geometric structures, transverse to the beam direction, are assumed to be infinite. Although many improvements of Fermi-Eyges theory were performed, e.g. by including additional correction factors $[2,32,33,48]$, they still suffer from the small angle and the homogeneity assumption. Comparisons with experimental data showed disadvantages in inhomogeneous phantoms [40].

A statistical simulation method for radiation transport problems is the Monte Carlo (MC) method [4]. It performs direct simulations of individual particle tracks which result from a random sequence of free flights and interaction events. In this way random histories are generated. If their number is large enough, macroscopic quantities can be obtained by averaging over the simulated histories. MC tools model physical processes very precisely and can handle arbitrary geometries without losing accuracy. Although they rank among the most accurate methods for predicting absorbed dose distributions, their highcomputation times limit their use in clinics. Due to the increase in computing power and decrease in hardware costs, $\mathrm{MC}$ techniques have recently become a growing field in radiotherapy [50]. Not only general-purpose MC codes are now publicly available, but also commercial MC treatment planning systems [11,49]. However, they have not yet gained widespread clinical use.

A different approach in the solution of radiation transport problems is deterministic calculations solving the linear BTE. In principle, its solution will give very accurate dose distributions comparable to that of MC simulations. The BTE can be analytically solved only in very simplified geometries, which is insufficient for clinical applications. Additionally, numerical solutions to the BTE require deterministic methods coping with a $6 \mathrm{D}$ phase space. Because of this and their simpler implementation, MC techniques have so far prevailed in the medical-physics community. Nevertheless, Börgers [7] argued that on certain accuracy conditions, deterministic methods could compete with MC calculations.

In this paper, we describe electron and photon transport in media by solving the generalized Fokker-Planck (GFP) approximation of the linear BTE [38]. For electron transport, this is an extension of the Fokker-Planck (FP) model, formulated in [24], to higher-order approximations in angle scattering. It should be stressed that this approach brings about several advantages: the BTE does not require any assumption on the geometry so that arbitrary heterogeneities are possible. Furthermore, we benefit from mathematical and physical approximation ansätze because they can be directly included in the differential equation. This avoids heuristic assumptions. In contrast to MC simulation, deterministic solutions do not suffer from statistical noise, and their resolution does not 
depend on the number of particles traversing a certain region. Moreover, the treatment planning problem can be formulated as a PDE-constrained optimization problem. This structure can be used to obtain additional information to speed-up the optimization $[18,19]$. This is hard to achieve by MC techniques.

Previous studies in deterministic methods for radiotherapy primarily concentrated on a combination of rigorous-analytic solutions and laboratory measurements (see pencil beam models above). Without explicitly using experimental data in their model, Huizenga and Storchi [27] presented the phase space evolution (PSE) model for electrons and subsequently applied it to multi-layered geometries [41]. Various improvements and extension to 3D beam dose calculations were performed [30,31]. However, 3D dose calculations showed disadvantages in computation times [34]. Moreover, PSE models used first-order discretizations in space which cannot compete with $\mathrm{MC}$ techniques [7]. By contrast, our access focuses on the continuous model of the Boltzmann equation discretized with high-order schemes.

First studies for deterministic dose calculations in charged-particle transport came from well-known procedures for numerical solutions to the transport equation of neutrons or photons. Several approximative models to the BTE have been developed. Each of these methods has its advantages and drawbacks [8]. Multi-group methods are sometimes used to discretize the energy domain [12]. This leads to a number of monoenergetic equations to be separately discretized in space and angle. Although discretizations in space are usually done by finite difference and finite element (FE) methods, the remaining angle domain is discretized by discrete ordinates [5,14]. Such an approach is implemented in the solver Attila [22]. Recently, 3D dose calculations for real clinical test cases were performed with Attila [53]. Results with similar accuracy as MC calculations were achieved in promising computation times.

Less frequent are angular FE approaches [11] seeking to reduce ray effects. Tervo et al. [51] extended FE discretizations to all variables in spatial, angular and energy domains so that no group cross sections were needed. Moreover, a detailed description of three coupled BTEs with FE discretizations of all variables was proposed in [6].

In this paper, the BTE is approximated by the continuous slowing down (CSD) method [36,39]. Scattering processes are modelled by GFP approximations [38] and compared to classical FP [44] solutions. Leakeas and Larsen [38] showed that scattering kernels with a sufficient amount of large-angle scattering yield inaccurate FP results. As an extension of the work in [38], we perform deterministic GFP simulations and investigate their behaviour in real applications. It is well known that electron scattering is dominantly forward peaked. Hence, many electron transport simulations used the classical FP approximation $[13,17,24]$. However, up to now, no comparisons to GFP dose computations have been done including realistic physical scattering cross sections. In our case, the latter are extracted from ICRU libraries [28]. We describe in detail how transport coefficients in the BTE can be computed from these scattering cross sections and compute GFP electron dose profiles in inhomogeneous geometries.

Even more challenging for GFP theory are scattering kernels including large angle scattering. We therefore investigate transport of photons in biological tissues with forward-peaked and large-angle scattering. Using test cases from [23] the radiative transport equation for GFP approximations up to order five is solved to determine reflectance and transmittance of light in biological tissues.

In the remaining paper, the following structure is presented: a short discussion of basic electron interactions with matter is given at the beginning. We describe a model for electron transport and review crucial steps of the GFP theory. In addition, we derive 
transport coefficients for the GFP equations from databases for electron scattering cross sections. In Section 3, a deterministic model for light propagation together with different scattering kernels is introduced. Discretization methods used in our GFP equations are studied in Section 4. In Section 5, we compute FP, GFP and discrete-ordinates' results for transmittance and reflectance of light by a slab. Moreover, we numerically compare FP, GFP and MC solutions for 5 and $10 \mathrm{MeV}$ electrons in homogeneous and heterogenous slab geometries. Section 6 gives the conclusions and outlooks. Appendix A contains explicit formulae for high-order polynomial operators. In Appendix B, we present the equations to be solved for the GFP coefficients.

\section{Deterministic model for electron transport}

\subsection{Physical interactions}

Electron beams are nowadays a widely spread tool in cancer therapy. Typical electron beams, provided by high-energy linear accelerators, range from 1 to $25 \mathrm{MeV}$. During irradiation of human tissue, electrons interact with matter through several competing mechanisms.

1. Elastic scattering. This is usually a non-radiative interaction between electrons and the atomic shell. Projectiles experience small deflections and lose little energy. High-energy electrons can also penetrate through atomic shells and are afterwards scattered at the bare nucleus without any energy loss. With kinetic energies above $1 \mathrm{keV}$, elastic scattering in water dominantly occurs in the forward direction [37].

2. Soft inelastic $e^{-}-e^{-}$scattering. Electrons interact with other electrons of the outer atomic shell which usually leads to excitation or ionization of the target particle. Here binding energies are only a few electron volts so that projectile electrons transfer little energy and are hardly deflected.

3. Hard inelastic $e^{-}-e^{-}$scattering. These collisions are determined by large transfer energies to the target electron. What 'large' exactly means is specified in MC codes by cutoff energies. In PENELOPE [46], for example, the default value of this simulation parameter is set to $1 \%$ of the maximum energy of all particles. As a consequence, the target electrons are ejected with larger scattering angles and higher kinetic energies (delta rays). They act as an additional source in the transport equation.

4. Bremsstrahlung. Owing to the electrostatic field of atoms, electrons are decelerated and hence emit bremsstrahlung photons. However, for energies below $1 \mathrm{MeV}$ this phenomenon can be neglected. Bremsstrahlung photons are not mainly emitted in the forward direction. The lower their kinetic energy the more isotropic their angle distribution becomes [35].

Evidently, there are more interaction processes such as ejection of Auger electrons or characteristic X-ray photons. But they are very unlikely in the energy range considered.

Although inelastic collisions are decisive for the energy transfer, the radiation damage in the patient strongly depends on the spatial distribution of electrons in their passage through matter. They dominantly undergo multiple scattering events with small deviations. However, single backward scattering events also occur frequently which leads to tortuous trajectories of electrons. To a big extent, such trajectories are due to elastic collisions. We therefore focus on very accurate and realistic simulation of elastic processes in our model. This is achieved by transport coefficients extracted from the ICRU 77 database [28]. Inelastic transport coefficients are obtained in the same way. 
Elastic and soft inelastic events lead to small energy loss. With kinetic energies above $1 \mathrm{keV}$, electrons are assumed to lose their energy continuously [21]. Because of this, we implement the CSD approximation to model energy loss by electrons. Hence, we neglect large energy loss fluctuations caused by hard inelastic collisions.

Bremsstrahlung effects are considered in our model in a very restricted way: only energy transfer of electrons after soft bremsstrahlung collisions is simulated by means of the radiative stopping power. However, the effect of hard photon emission as well as the transport of photons is disregarded so far.

\subsection{The GFP approximation for electrons}

The motion of particles can be described by a $6 \mathrm{D}$ phase space $(\underline{r}, E, \underline{\Omega}) \in\left(V \times I \times S^{2}\right)$ with

$$
\begin{aligned}
& \text { spatial variable } \underline{r}=\left(x_{1}, x_{2}, x_{3}\right) \in V \subset \mathbb{R}^{3} \text { open and bounded, } \\
& \text { energy variable } E \in I=\left[E_{\min }, E_{\max }\right] \subset \mathbb{R}, \\
& \text { direction variable } \underline{\Omega}=\left(\Omega_{1}, \Omega_{2}, \Omega_{3}\right) \in S^{2} \subset \mathbb{R}^{3} \text { unit sphere } \\
& \quad=\left(\sqrt{1-\mu^{2}} \cos (\phi), \sqrt{1-\mu^{2}} \sin (\phi), \mu\right), \quad \mu=\cos (\theta)
\end{aligned}
$$

If particle transport takes place in an isotropic and homogeneous medium in which interaction processes are Markovian and particles do not interact with themselves, their distribution can be described by the unique solution of the time-dependent linear BTE. For practical applications in radiotherapy, we are faced with issues such as distributions of the deposited energy in biological tissues or penetration depths of the beam. For many purposes it is, therefore, sufficient to know the steady solution:

$$
\sigma_{a} \Psi(\underline{r}, E, \underline{\Omega})+\underline{\Omega} \cdot \nabla \Psi(\underline{r}, E, \underline{\Omega})=L_{\mathrm{B}} \Psi(\underline{r}, E, \underline{\Omega})
$$

with

$$
\Psi(\underline{r}, E, \underline{\Omega})=\Psi_{b}(\underline{r}, E, \underline{\Omega}) \quad \text { for all } \underline{r} \in \partial V, \underline{\Omega} \cdot \underline{n}<0, E \in I,
$$

where $\Psi(\underline{r}, E, \underline{\Omega})$ is called angular flux and denotes the distribution of particles travelling in direction $\underline{\Omega}$. Boundary conditions (BC) are imposed on the angular flux depending on the incident beam. Absorption of particles is described by the absorption cross section $\sigma_{a}$. The right-hand-side

$$
L_{\mathrm{B}} \Psi(\underline{r}, E, \underline{\Omega}):=\int_{0}^{\infty} \int_{4 \pi} \sigma_{s}\left(E^{\prime}, E, \underline{\Omega} \cdot \underline{\Omega}^{\prime}\right) \Psi\left(\underline{r}, E^{\prime}, \underline{\Omega}^{\prime}\right) \mathrm{d} \Omega^{\prime} \mathrm{d} E^{\prime}-\Sigma_{s}(E) \Psi(\underline{r}, E, \underline{\Omega})
$$

is known as linear Boltzmann Operator which describes scattering. Its integral contains the differential scattering cross section (DSCS) $\sigma_{s}\left(E^{\prime}, E, \underline{\Omega} \cdot \underline{\Omega}^{\prime}\right)$ characterizing interaction mechanisms in which particles are deflected. The dot product $\underline{\Omega} \cdot \underline{\Omega^{\prime}}=\cos \left(\theta_{0}\right)=\mu_{0}$ indicates that the scattering probability only depends on the scattering angle. This implies that the deflection of scattered particles is axially symmetrical around the direction of incidence $\underline{\Omega}^{\prime}$. Integrating the scattering kernel $\sigma_{s}\left(E^{\prime}, E, \underline{\Omega} \cdot \underline{\Omega}^{\prime}\right)$ over all angles and 
energies, one gets the total DSCS (TDSCS)

$$
\Sigma_{s}(E)=2 \pi \int_{0}^{\infty} \int_{-1}^{1} \sigma_{s}\left(E^{\prime}, E, \mu_{0}\right) \mathrm{d} \mu_{0} \mathrm{~d} E^{\prime}
$$

The angle and energy integral in the Boltzmann operator is the main difficulty in its numerical solution. That is why an important aim is to develop accurate approximations. However, up to now, there is no predominant method used for all types of particles. In fact, depending on specific particle properties, one has to choose an appropriate approximation.

One crucial property of elastic DSCSs in water is a sharp peak in the forward direction [29]. To express this mathematically, we define the positive $n$th scattering transport coefficient (STC)

$$
\xi_{n}(E):=2 \pi \int_{0}^{\infty} \int_{-1}^{1}\left(1-\mu_{0}\right)^{n} \sigma_{s}\left(E^{\prime}, E, \mu_{0}\right) \mathrm{d} \mu_{0} \mathrm{~d} E^{\prime}, \quad \text { for all } n \geq 0
$$

and assume that as $n$ increases, the coefficients $\xi_{n}$ fall off sufficiently fast, i.e.

$$
\xi_{n+1}(E) \ll \xi_{n}(E), \quad \text { for all } n \geq 0 \text { and } E \in I .
$$

Additionally, elastic scattering often entails a small energy loss. Therefore, the first approximation is the expansion of the scattering kernel around $\mu_{0}=1$ and $E=E^{\prime}$. In this way, Pomraning [44] showed that the already known FP operator is the lowest-order asymptotic limit of the integral operator $L_{\mathrm{B}}$. In [39], this FP operator is derived as a first order angle approximation to $L_{\mathrm{B}}$ :

$$
\text { FP operator } L_{\mathrm{FP}}:=\left(\xi_{1} / 2\right) L
$$

where

$$
L_{\mathrm{B}} \Psi(\underline{\Omega})=L_{\mathrm{FP}} \Psi(\underline{\Omega})+\mathcal{O}(\varepsilon), \quad \text { for } \varepsilon \ll 1 .
$$

As the spherical Laplace-Beltrami operator

$$
L=\left[\frac{\partial}{\partial \mu}\left(1-\mu^{2}\right) \frac{\partial}{\partial \mu}+\frac{1}{1-\mu^{2}} \frac{\partial^{2}}{\partial \phi^{2}}\right], \quad \text { with } \mu=\cos (\theta),
$$

is differential in angle, the non-local integral Boltzmann operator $L_{\mathrm{B}}$ is now approximated by a local differential operator. The crucial point is that an integro-differential equation is transformed into a partial differential equation. Although discretizations of differential equations often lead to large linear systems, their numerical effort turns out to be much lower. This is due to the local character of differential equations, which bring along much sparser matrices.

Pomraning's resulting FP equation for particle transport in an isotropic medium yields

$$
\sigma_{a} \Psi(\underline{r}, E, \underline{\Omega})+\underline{\Omega} \cdot \nabla \Psi(\underline{r}, E, \underline{\Omega})=\frac{\xi_{1}(E)}{2} L \Psi(\underline{r}, E, \underline{\Omega})+\frac{\partial(S(\underline{r}, E) \Psi(\underline{r}, E, \underline{\Omega}))}{\partial E},
$$


where $S(\underline{r}, \varepsilon)$ is called stopping power defined by

$$
S(\underline{r}, E)=\int_{0}^{\infty} \int_{4 \pi}\left(E-E^{\prime}\right) \sigma_{s}\left(E^{\prime}, E, \underline{\Omega} \cdot \underline{\Omega^{\prime}}\right) \mathrm{d} \Omega \mathrm{d} E^{\prime} .
$$

The standard FP approximation is a frequently used method to describe transport processes in media with highly forward-peaked scattering. Comparisons to real data, however, reveal that many scattering processes of interest contain a small but sufficient amount of largeangle scattering. To gain higher-order asymptotic approximations to $L_{\mathrm{B}}$, one could expand $L_{\mathrm{B}}$ to a

$$
\text { Polynomial operator } L_{P n}:=\sum_{m=1}^{n} a_{n, m} L^{m},
$$

with

$$
a_{n, m} \in \mathcal{O}\left(\xi_{m}\right)=\mathcal{O}\left(\varepsilon^{m-1}\right) \text { and } L_{\mathrm{B}} \Psi(\underline{\Omega})=L_{P n} \Psi(\underline{\Omega})+\mathcal{O}\left(\varepsilon^{n}\right), \text { for all } n \geq 1 .
$$

However, Leakeas and Larsen [39] showed that eigenvalues of $L_{P n}$ might become positive so that the angular flux could become infinite. This served as a motivation for them to develop asymptotically equivalent operators to $L_{P n}$, which remain stable and preserve certain eigenvalues of $L_{\mathrm{B}}$. We have summarized the details of the computation in the appendix. We end up with GFP equations which incorporate large-angle scattering and are, therefore, more accurate than the conventional FP equation:

$$
\sigma_{a} \Psi(\underline{r}, E, \underline{\Omega})+\underline{\Omega} \cdot \nabla \Psi(\underline{r}, E, \underline{\Omega})=L_{\mathrm{GFP}_{n}} \Psi(\underline{r}, E, \underline{\Omega})+\frac{\partial(S(\underline{r}, E) \Psi(\underline{r}, E, \underline{\Omega}))}{\partial E} .
$$

For positive coefficients $\alpha_{i}(E), \beta_{i}(E)$ and $m \in \mathbb{N}$, the GFP operators are defined by

$$
L_{\mathrm{GFP}_{2 m}}:=\sum_{i=1}^{m} \alpha_{i}(E) L\left(I-\beta_{i}(E) L\right)^{-1} \quad \text { and } \quad L_{\mathrm{GFP}_{2 m+1}}:=L_{\mathrm{GFP}_{2 m}}+\alpha_{m+1}(E) L .
$$

\subsection{Determination of physical quantities}

The fundamental part in GFP theory is based on the assumption of forward-peakedness (Equation (1)). Its expected accuracy strongly depends on the behaviour of transport coefficients $\xi_{n}(E)$, which are different for the various projectiles and materials. The ICRU Report 77 provides differential cross sections for elastic and inelastic scattering of electrons and positrons for different materials and energies between 50 and $100 \mathrm{MeV}$ [28]. To obtain transport coefficients $\xi_{n}(E)$, we use these cross sections and proceed in the following way:

(a) The ELSEPA code system, distributed with the report, calculates elastic and inelastic angular differential cross sections for a fixed energy $E$,

$$
\sigma^{\mathrm{el}, \text { inel }}\left(E, \mu_{0}\right)=\int_{0}^{\infty} \sigma_{s}^{\mathrm{el}, \text { inel }}\left(E^{\prime}, E, \mu_{0}\right) \mathrm{d} E^{\prime}
$$

in tabulated form for discrete $\mu_{0}$ and $\sigma^{\text {el,inel }}\left(E, \mu_{0}\right)$. For a predetermined set of energy values between 50 and $100 \mathrm{MeV}$, data for $\sigma^{\text {el,inel }}\left(E, \mu_{0}\right)$ are extracted from these files. 
(b) With this we calculate the $n$th transport coefficient for a fixed energy $E$,

$$
\xi_{n}^{\mathrm{el}, \text { inel }}(E)=2 \pi \mathcal{N} \int_{-1}^{1}\left(1-\mu_{0}\right)^{n} \sigma^{\mathrm{el}, \text { inel }}\left(E, \mu_{0}\right) \mathrm{d} \mu_{0}, \quad \text { with } \mu_{0}=\cos \left(\theta_{0}\right)
$$

via numerical integration of the tabulated cross sections $\sigma^{\text {el,inel }}\left(E, \mu_{0}\right)$ by means of the trapezodial rule. Additionally, we multiply the result by the molecular density of the transmitted matter

$$
\mathcal{N}=N_{A} \frac{\rho}{A},
$$

where $N_{A}$ is Avogadro's number, $\rho$ the mass density of the material and $A$ its molar mass.

(c) Again, all computed results of $\xi_{n}^{\text {el,inel }}(E)$ are stored and used as a look-up table. To obtain the $n$th transport coefficient at the desired energy $E$, these tabulated data are linear interpolated.

Finally, we use the following transport coefficient in our equation:

$$
\xi_{n}(E):=2 \pi \mathcal{N} \int_{-1}^{1}\left(1-\mu_{0}\right)^{n}\left(\sigma^{\mathrm{el}}\left(E, \mu_{0}\right)+\sigma^{\mathrm{inel}}\left(E, \mu_{0}\right)\right) \mathrm{d} \mu_{0} .
$$

Figure 1 illustrates the electron transport coefficients of different order in liquid water. Except for the 0th coefficient, every other coefficient is strictly monotonically decreasing. For $E \geq 10^{-3}, \xi_{1}$ is always bigger than $\xi_{2}$ but, as $E$ decreases, their deviation reduces more

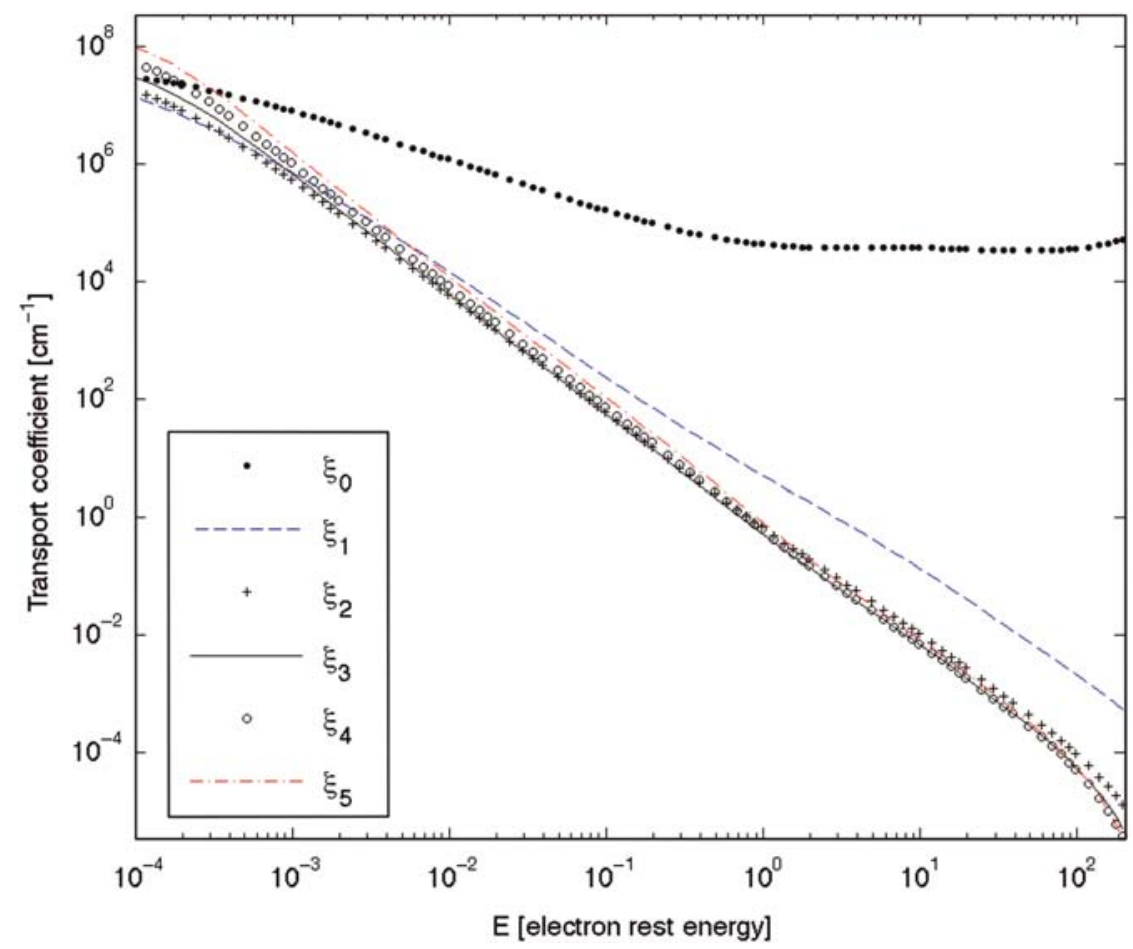

Figure 1. Comparison of STC in liquid water for energies between 50 and $100 \mathrm{MeV}$. 
and more. Unfortunately, for increasing $n \geq 2$ the difference between two consecutive $\xi_{n}$ is so small that the assumption of forward-peakedness is not fulfilled. The inelastic transport coefficients are much smaller than elastic transport coefficients for $n \geq 1$. The higher the order of the transport coefficient, the larger is the difference between elastic and inelastic ones.

Our stopping power in Equation (7) is equivalent to the physical total stopping power as the sum of collision and radiative stopping powers. For different materials both are directly included in the files of the ICRU database. Hence, we do a linear interpolation to get the stopping power $S(E)$ at the desired energy $E$.

\section{Deterministic model for light propagation}

\subsection{The GFP equation for grey photons}

Many medical applications such as cancer treatment or optical imaging of tumours make use of propagation of laser light in biological tissues. Its behaviour is determined by the solution of the steady grey radiative transport equation

$$
\sigma_{a} \Psi(\underline{r}, \underline{\Omega})+\underline{\Omega} \cdot \nabla \Psi(\underline{r}, \underline{\Omega})=\mu_{s}\left[\int_{4 \pi} \sigma_{s}\left(\underline{\Omega} \cdot \underline{\Omega^{\prime}}\right) \Psi(\underline{r}, \underline{\Omega}) \mathrm{d} \Omega^{\prime}-\Psi(\underline{r}, \underline{\Omega})\right],
$$

with

$$
\Psi(\underline{r}, \underline{\Omega})=\Psi_{b}(\underline{r}, \underline{\Omega}), \quad \text { for all } \underline{r} \in \partial V, \underline{\Omega} \cdot \underline{n}<0 \text {. }
$$

Similar to 2.2, one can derive GFP approximations to the radiative transport equation in a straightforward way. The intensity $\Psi(\underline{r}, \underline{\Omega})$ describes the radiation power flowing in direction $\Omega$ which is influenced by scattering and absorption coefficients $\sigma_{a}$ and $\mu_{s}$. More important is the scattering kernel $\sigma_{s}\left(\underline{\Omega} \cdot \underline{\Omega}^{\prime}\right)$. It is also characteristic for biological tissues that this kernel has a sharp peak at $\underline{\Omega} \cdot \underline{\Omega}^{\prime}=1$. For its simulation, mathematically simple scattering kernels with a free parameter are used.

\subsection{Models for scattering kernels}

One often cited scattering kernel is the (single) Henyey-Greenstein (HG) kernel [25] defined by

$$
\sigma_{s}^{\mathrm{HG}}\left(\mu_{0}\right)=\frac{\sum_{s}^{\mathrm{HG}}}{2 \pi} f_{\mathrm{HG}}\left(\mu_{0}\right)
$$

where

$$
f_{\mathrm{HG}}\left(\mu_{0}\right):=\frac{1-g^{2}}{2\left(1-2 g \mu_{0}+g^{2}\right)^{3 / 2}}, \quad \text { for } g \in(-1 ; 1),
$$

is the corresponding phase function, and $\Sigma_{s}^{\mathrm{HG}}$ the TDSCS. The single parameter $g$ determines the amount of small- and large-angle scattering. If $g \approx 1, \sigma_{s}^{\mathrm{HG}}$ is not only strongly forward peaked but also includes large-angle scattering. Its value depends on the irradiated tissue. For biological tissues, typical values for $g$ are around 0.9 (human blood: $g=0.99$, human dermis: $g=0.81[9])$. Expanding $f_{\mathrm{HG}}\left(\mu_{0}\right)$ in Legendre polynomials 
gives expressions for $\xi_{n}$ depending only on $g$ :

$$
\begin{gathered}
\xi_{1}=1-g, \\
\xi_{2}=\frac{4}{3}-2 g+\frac{2}{3} g^{2}, \\
\xi_{3}=2-\frac{18}{5} g+2 g^{2}-\frac{2}{5} g^{3}, \\
\xi_{4}=\frac{16}{5}-\frac{32}{5} g+\frac{32}{7} g^{2}-\frac{8}{5} g^{3}+\frac{8}{35} g^{4}, \\
\xi_{5}=\frac{16}{3}-\frac{80}{7} g+\frac{200}{21} g^{2}-\frac{40}{9} g^{3}+\frac{8}{7} g^{4}-\frac{8}{63} g^{5} .
\end{gathered}
$$

This is all we need in order to calculate $\alpha_{i}$ and $\beta_{i}$ for $\mathrm{GFP}_{2}-\mathrm{GFP}_{5}$. It turns out that they remain positive, without exception.

To control large-angle and forward-peaked scattering, a linear combination of forward and backward $\mathrm{HG}$ phase functions was introduced [23]. For real constants $g_{1} \in$ $(-1 ; 0], g_{2} \in[0 ; 1), b \in[0 ; 1]$ and the phase function

$$
f_{\mathrm{DHG}}\left(\mu_{0}\right):=b f_{\mathrm{HG}}\left(\mu_{0}, g_{1}\right)+(1-b) f_{\mathrm{HG}}\left(\mu_{0}, g_{2}\right),
$$

the double HG scattering kernel is defined by

$$
\sigma_{s}^{\mathrm{DHG}}\left(\mu_{0}\right)=\frac{\sum_{s}^{\mathrm{DHG}}}{2 \pi} f_{\mathrm{DHG}}\left(\mu_{0}\right)
$$

An indicator for the amount of forward or backward scattering is the constant $b$. Setting $b=0$, the backward scattering phase function $f_{\mathrm{HG}}\left(\mu_{0}, g_{1}\right)$ vanishes, whereas $b=1$ reduces $f_{\mathrm{DHG}}\left(\mu_{0}\right)$ to the single $\mathrm{HG}$ phase function $f_{\mathrm{HG}}\left(\mu_{0}, g_{1}\right)$. This provides an opportunity to adapt it to the material of interest.

Analogous to the above procedure for the single $\mathrm{HG}$ phase function, one can conclude that

$$
\sigma_{s n}=b\left(g_{1}^{n}-g_{2}^{n}\right)+g_{2}^{n},
$$

from which all STCs $\xi_{n}$ can be calculated.

\section{Numerics for GFP}

\subsection{Discretization of differential GFP equations}

Replacing the right-hand-side of the Boltzmann equation by the $\mathrm{GFP}_{2}$ approximation operator yields

$$
\begin{aligned}
& S(\underline{r}, E) \frac{\partial \Psi(\underline{r}, E, \underline{\Omega})}{\partial E}+\sigma_{a} \Psi(\underline{r}, E, \underline{\Omega})+\underline{\Omega} \cdot \nabla \Psi(\underline{r}, E, \underline{\Omega}) \\
& \quad=\alpha(E) L(I-\beta(E) L)^{-1} \Psi(\underline{r}, E, \underline{\Omega})+\Psi(\underline{r}, E, \underline{\Omega}) \frac{\partial S(\underline{r}, E)}{\partial E} .
\end{aligned}
$$


To solve Equation (18), we restate it by setting

$$
\Psi^{(0)}=\Psi(\underline{r}, E, \underline{\Omega}) \quad \text { and } \quad \Psi^{(1)}=(I-\beta(E) L)^{-1} \Psi^{(0)},
$$

so that it becomes

$$
\begin{gathered}
S(\underline{r}, E) \frac{\partial \Psi^{(0)}(\underline{r}, E, \underline{\Omega})}{\partial E}+\sigma_{a} \Psi^{(0)}(\underline{r}, E, \underline{\Omega})+\underline{\Omega} \cdot \nabla \Psi^{(0)}(\underline{r}, E, \underline{\Omega}) \\
=\alpha(E) L \Psi^{(1)}(\underline{r}, E, \underline{\Omega})+\Psi^{(0)}(\underline{r}, E, \underline{\Omega}) \frac{\partial S(\underline{r}, E)}{\partial E}, \\
(I-\beta(E) L) \Psi^{(1)}(\underline{r}, E, \underline{\Omega})=\Psi^{(0)}(\underline{r}, E, \underline{\Omega}) .
\end{gathered}
$$

These equations form a coupled system of second-order differential equations with the angular momentum operator $L$. Solving this system requires differencing schemes for the space and angle variable. Initial and $\mathrm{BC}$ are imposed on $\Psi^{(0)}$.

The asymptotic GFP analysis transformed the original BTE into a new type of equation which requires an additional condition to the energy variable:

$$
\Psi^{(0)}\left(\underline{r}, E_{\infty}, \underline{\Omega}\right)=0
$$

where $E_{\infty}$ denotes a large cutoff energy. In the numerical simulations, it should be bigger than the energy of all particles from the incoming beam.

A simplified model to be studied is that of a plate which is infinitely extended in $x$ - and $y$-direction with a thickness $d$ in $z$-direction. Owing to symmetry reasons

- the angular flux is independent of $x$ - and $y$-direction and

- its direction of motion $\underline{\Omega}$ only depends on $\theta$.

That is why the initial $6 \mathrm{D}$ problem has now been reduced to a $3 \mathrm{D}$ one. Although it seems that we only describe a 1D object in space, one should not forget the fact that our slab still remains 3D. From the mathematical point of view, the symmetry of this model, however, leads to a $1 \mathrm{D}$ problem in space which decreases computational costs.

In slab geometry, the aforementioned system reduces to

$$
\begin{gathered}
S(\underline{r}, E) \frac{\partial \Psi^{(0)}(z, E, \mu)}{\partial E}=\alpha(E) L_{\mu} \Psi^{(1)}(z, E, \mu)+\Psi^{(0)}(\underline{r}, E, \underline{\Omega}) \frac{\partial S(\underline{r}, E)}{\partial E} \\
-\sigma_{a} \Psi^{(0)}(z, E, \mu)-\frac{\partial \Psi^{(0)}(z, E, \mu)}{\partial z} \cdot \mu, \\
\left(I-\beta(E) L_{\mu}\right) \Psi^{(1)}(z, E, \mu)=\Psi^{(0)}(z, E, \mu),
\end{gathered}
$$

where the $1 \mathrm{D}$ angular momentum operator $L_{\mu}$ is defined by

$$
L_{\mu}:=\frac{\partial}{\partial \mu}\left[\left(1-\mu^{2}\right) \frac{\partial}{\partial \mu}\right] .
$$

We solve this system in two steps:

(1) to obtain the solution $\Psi^{(1)}(z, E, \mu)$ to Equation (23),

(2) to plug $\Psi^{(1)}(z, E, \mu)$ in Equation (22) and solve the resulting differential equation. 
To achieve accurate results and lower computation times, a high-order scheme was implemented [42]:

$$
\begin{gathered}
\tilde{L}_{\mu} \Psi^{(1)}\left(\mu_{j}\right)=\frac{1}{w_{j}}\left[D_{j+1 / 2} \frac{\Psi_{j+1}^{(1)}-\Psi_{j}^{(1)}}{\mu_{j+1}-\mu_{j}}-D_{j-1 / 2} \frac{\Psi_{j}^{(1)}-\Psi_{j-1}^{(1)}}{\mu_{j}-\mu_{j-1}}\right], \\
D_{j+1 / 2}=D_{j-1 / 2}-2 \mu_{j} w_{j},
\end{gathered}
$$

with

$$
D_{1 / 2}=0=D_{M+1 / 2}
$$

where $\mu_{j}$ are abscissas for a Gauss-Legendre quadrature rule with weights $w_{j}$.

With knowledge of the already calculated explicit values $\Psi_{i, j}^{(1)}$, the right-hand-side of Equation (22) reduces to a single $\Psi^{(0)}$ dependence. The resulting partial differential equation is discretized with finite differences in the $z$-direction. Hence, we end up with an ordinary differential equation in the energy variable $E$. Its solution is obtained by the embedded 2nd/3rd order Runge-Kutta MATLAB solver ode23 solving from the initial condition in Equation (21) backward in energy to $E=0$. The remaining discretizations are of first order in $z$ and of second order in $\mu$.

Higher-order GFP equations are discretized and solved analogously. However, due to more frequent occurrence of $L_{\mu}$ and $\left(I-\beta(E) L_{\mu}\right)$, the right-hand-side of Equation (22) becomes more involved and more systems have to be solved.

\section{Numerical results}

\subsection{Slab geometry: HG kernel}

First, we neglect absorption and start with a simpler form of the GFP equation

$$
\underline{\Omega} \cdot \nabla \Psi(\underline{r}, \underline{\Omega})=L_{\mathrm{GFP}_{n}} \Psi(\underline{r}, \underline{\Omega}) \text {. }
$$

This is solved in slab geometry with the HG scattering kernel from Equation (10) for selected values of the anisotropy factor $g$. Symmetry properties mentioned above yield the following boundary value problem (exemplarily stated for $\mathrm{GFP}_{2}$ ):

$$
\mu \frac{\partial \Psi^{(0)}(z, \mu)}{\partial z}=\alpha L_{\mu} \Psi^{(1)}(z, \mu) \quad\left(I-\beta L_{\mu}\right) \Psi^{(1)}(z, \mu)=\Psi^{(0)}(z, \mu)
$$

with BC

$$
\Psi^{(0)}(0, \mu)=10^{5} \cdot \mathrm{e}^{-10(1-\mu)^{2}}, 1 \geq \mu>0, \quad \text { and } \quad \Psi^{(0)}(d, \mu)=0,-1 \leq \mu<0 .
$$

For $g=0.8$ and 0.95 , results were computed by time marching with an adaptive RungeKutta solver until a steady state was reached. Incoming photons moving in positive $z$-direction at $z=0$ are simulated by narrow Gaussian peaks around $\mu=1$. Corresponding graphs illustrate steady solutions and use penetration depth in centimetres as $x$ - and $\int_{-1}^{1} \Psi(z, \mu) \mathrm{d} \mu$ in $1 /\left(\mathrm{J} \mathrm{cm}^{2} \mathrm{~s}\right)$ as $y$-axis. The latter quantity is sometimes called energy density and is related to the dose. Discretization parameters in $z$ (110 points) and in $\mu$ (64 points) directions were chosen large enough to reach convergence. Figures 2 and 3 


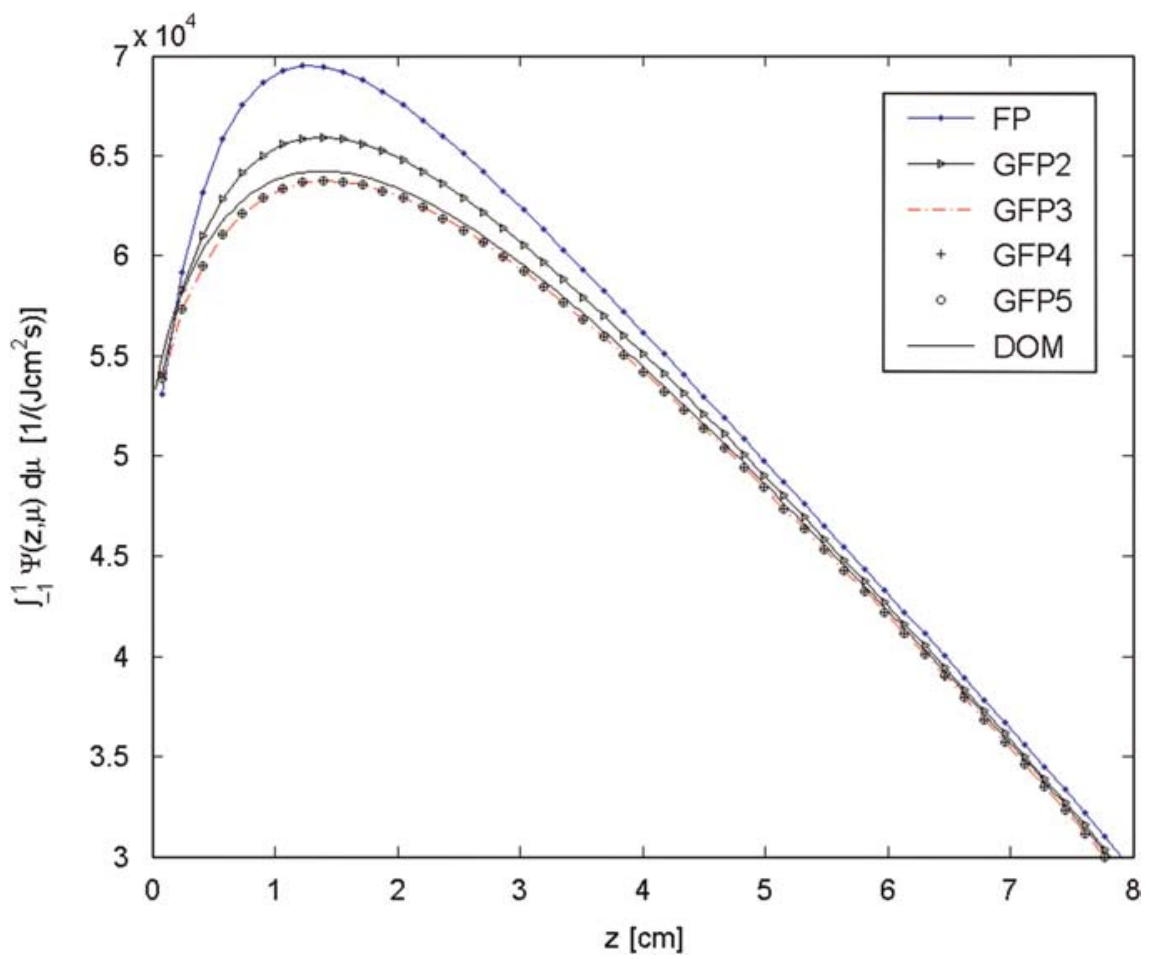

Figure 2. HG kernel in slab geometry with $g=0.80$. The difference between $\mathrm{GFP}_{3}-\mathrm{GFP}_{5}$ lines is so small that they almost agree here.

additionally show converged transport solutions generated by a discrete ordinates method (DOM); which we use as benchmark in the following.

Each distribution forms a monotonically increasing function until it reaches a maximum and strictly decreases afterwards. There is a large difference between FP, $\mathrm{GFP}_{2}$ and the other GFP approximations. However, $\mathrm{GFP}_{3}-\mathrm{GFP}_{5}$ are hardly distinguishable. For increasing values of $g$, this discrepancy between $\mathrm{GFP}_{3}$ and $\mathrm{GFP}_{5}$ becomes bigger, whereas results for $\mathrm{GFP}_{4}$ and $\mathrm{GFP}_{5}$ show no distance at all. When it comes to the DOM curve, we observe that $\mathrm{FP}$ and $\mathrm{GFP}_{2}$ give poor approximations for small penetration depths. However, $\mathrm{GFP}_{3-5}$ give quite good solutions throughout the whole penetration range.

\subsection{Single slab: light propagation in biological tissues}

González-Rodríguez and Kim [23] studied light propagation in tissues including both forward-peaked and large-angle scattering. They examined several approximation methods and especially implemented $\mathrm{GFP}_{2}$. We want to augment this with results up to $\mathrm{GFP}_{5}$ and compare the resulting simulations with the transport solution. We focus on the following problem:

$$
\sigma_{a} \Psi(z, \mu)+\mu \frac{\partial \Psi(z, \mu)}{\partial z}=\mu_{s}\left[\int_{4 \pi} \sigma_{s}\left(\underline{\Omega} \cdot \underline{\Omega^{\prime}}\right) \Psi\left(z, \underline{\Omega^{\prime}}\right) \mathrm{d} \Omega^{\prime}-\Psi(z, \mu)\right],
$$

with $\mathrm{BC}$

$$
\Psi(z=0, \mu)=\mathrm{e}^{-10(1-\mu)^{2}}, 1 \geq \mu>0, \quad \text { and } \quad \Psi(z=d, \mu)=0,-1 \leq \mu<0 .
$$




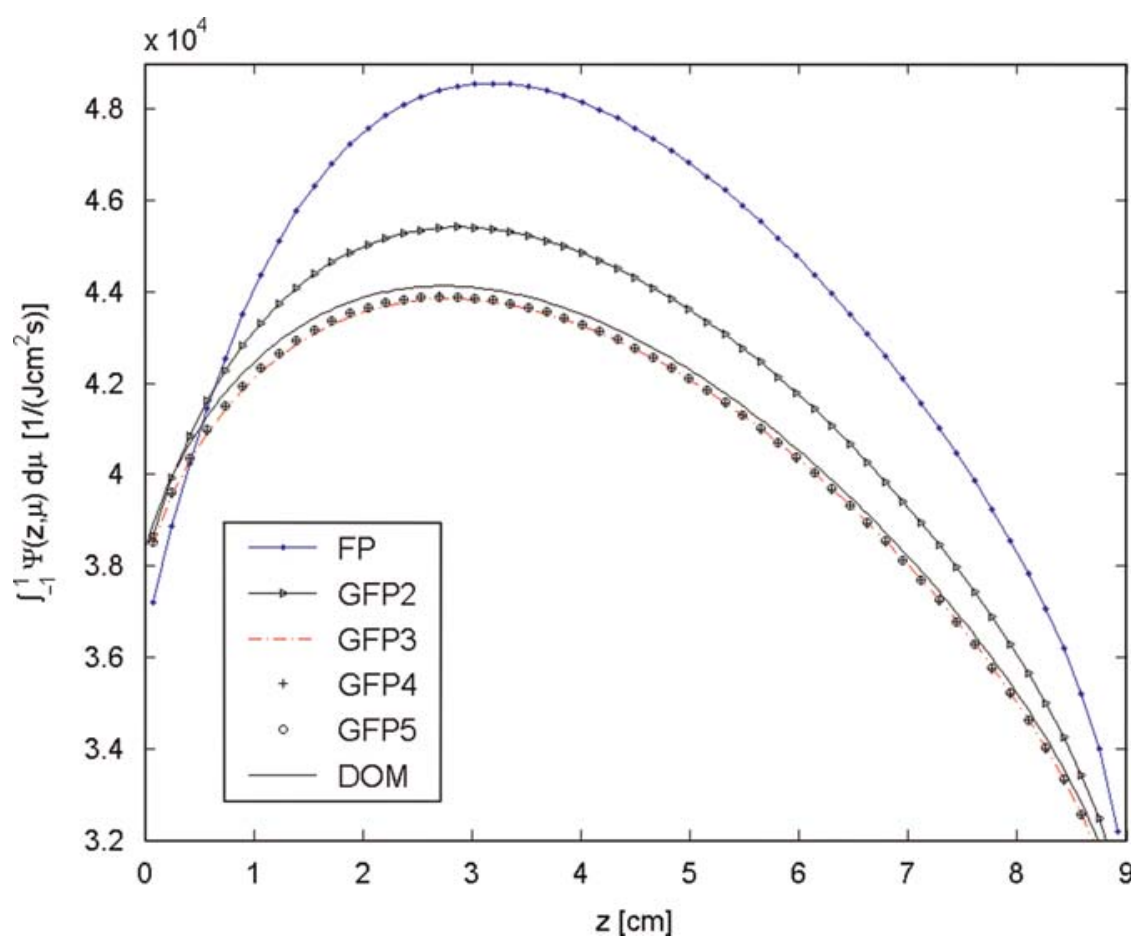

Figure 3. HG kernel in slab geometry with $g=0.95$. Solutions for $\mathrm{GFP}_{3}-\mathrm{GFP}_{5}$ already overlap.

It is a slab geometry with a thickness of $d=2 \mathrm{~mm}$ disregarding any time dependence. Its solution enables to compute reflectance $R(\mu)$ and transmittance $T(\mu)$ defined by

$$
R(\mu):=\Psi(\mu, 0) \quad-1 \leq \mu<0 \quad T(\mu):=\Psi(\mu, d) \quad 1 \geq \mu>0 .
$$

\subsubsection{Single Henyey-Greenstein kernel}

In the first run, Equation (28) was solved with discretizations of 64 points in angle and 80 points in space using GFP approximations with the HG DSCS $\sigma_{s}=\sigma_{s}^{\mathrm{HG}}$. Further constants were set to

$$
g=0.98, \quad \sigma_{a}=0.01 \mathrm{~mm}^{-1}, \quad \mu_{s}=50 \mathrm{~mm}^{-1} .
$$

Reflectance. Starting at $R(-1) \approx 0.23$, the reflectance slightly increases and attains its maximum at $\mu \approx-0.5$ (Figure 4 ). Although in this interval GFP data show discrepancies among each other, their results are accurate and $\mathrm{GFP}_{3}$ gives the best approximation. For $\mu>-0.5$, the transport solution DOM hunches down more than GFP functions and hence, the error increases rapidly. Surprisingly, for $\mu \gtrsim-0.25, \mathrm{GFP}_{3}$-reflectance values are closer to DOM than those of $\mathrm{GFP}_{5}$. Throughout the whole interval, FP values give a very poor approximation.

Transmittance. It is almost a straight line, only bending for small $\mu$. In contrast to the reflectance, a more or less constant distance to the transport solution is always sustained. To the eye, there are no differences between all GFP simulations in a wide range. Only for 

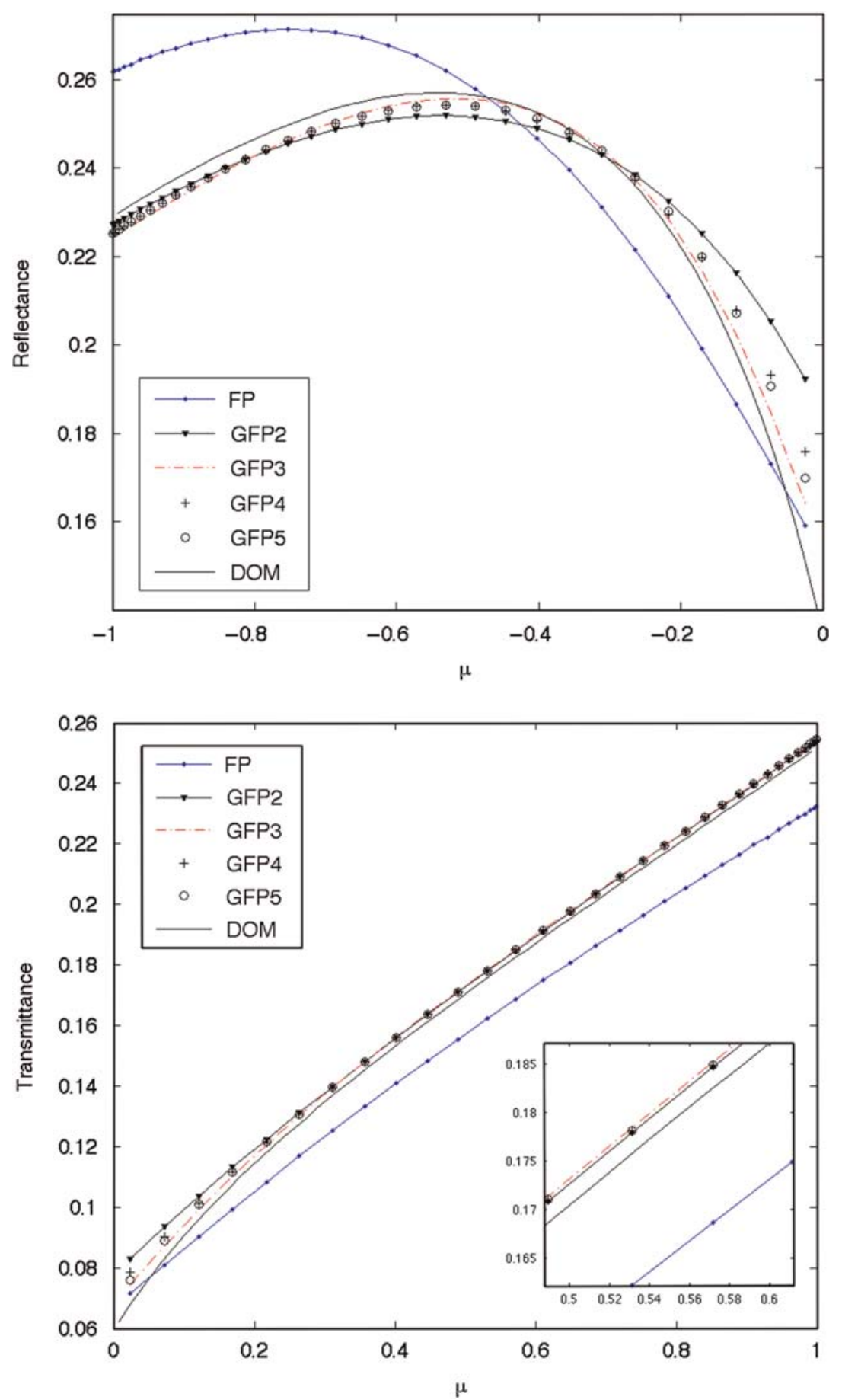

Figure 4. Single HG with $g=0.98$ : reflectance and transmittance of liver tissue arising from a slab geometry with thickness $d=2 \mathrm{~mm}$ and conditions as in Equation (28). Transmittance is plotted in a semi-logarithmic scale. 
small $\mu$, the functions start to deviate and $\mathrm{GFP}_{3}$ data give best results, whereas FP is inaccurate again.

\subsubsection{Double Henyey-Greenstein kernel}

Taking the amount of large-angle scattering in biological tissues into account, GonzálezRodríguez and Kim applied the double HG DSCS to simulate transmittance and reflectance in liver tissue. The following fit parameters were used:

$$
g_{1}=0.85, g_{2}=-0.34, \quad b=0.86,
$$

where $g_{1}=0.85$ provides a forward peak which is not very sharp. In addition, the combination of $g_{2}=-0.34$ and $b=0.86$ contains a significant amount of large-angle scattering which leads to increasing STCs:

$$
\xi_{1}=0.3166, \quad \xi_{2}=0.3916, \quad \xi_{3}=0.6058, \quad \xi_{4}=1.0075, \quad \xi_{5}=1.7388 .
$$

In this case, our fundamental assumption is not valid which could negatively affect our approximations. Moreover, it is important to emphasize that simulations for $\mathrm{GFP}_{3}-\mathrm{GFP}_{5}$ ran with some negative coefficients $\alpha_{i}, \beta_{i}$. Nevertheless, our code gave reasonable results plotted in Figure 5 for discretization parameters of 64 points in $\mu$ and 70 points in $z$-direction.

Reflectance. Figure 5 shows 'bump head' functions similarly shaped to those of the single HG kernel. The $x$-coordinates of their maxima are, however, shifted to the right. Moreover, for large $\mu$, different GFP approximations do not match as well as they do in Figure 4. In contrast to the single $\mathrm{HG}$ kernel, $\mathrm{GFP}_{3}$ data give a poor approximation, whereas $\mathrm{GFP}_{5}$ is the best one among all shown here. Only for $\mu \approx 0, \mathrm{GFP}_{2}$ is not able to match $\mathrm{GFP}_{5}$. As expected, FP gives even worse results than for the single HG kernel.

Transmittance. This time our transport solution DOM is more peaked at $\mu \approx 1$. Nevertheless, GFP gives more accurate results than in Figure 4. A comparison between the two best approximations $\mathrm{GFP}_{3}$ and $\mathrm{GFP}_{5}$ yields small differences which enlarge near $\mu \approx 0$. However, the classical FP deviates from our benchmark to a big extent.

Owing to the contribution of large-angle scattering, numerical computations with the double HG kernel are more challenging and, in fact, give GFP coefficients which contradict our assumptions. Nevertheless, the GFP results plotted above approximate the transport solution very well and are much more precise than those of the FP calculations.

\subsection{Slab geometry: electron propagation in biological tissues}

For dose calculations, the following GFP equation is to be solved (examplarily stated for $\left.\mathrm{GFP}_{2}\right)$ :

$$
\begin{aligned}
& \sigma_{a} \Psi^{(0)}(z, E, \mu)+\frac{\partial \Psi^{(0)}(z, E, \mu)}{\partial z} \cdot \mu=\alpha L_{\mu} \Psi^{(1)}(z, E, \mu)+\frac{\partial\left(S(z, E) \Psi^{(0)}(z, E, \mu)\right)}{\partial E} \\
& \left(I-\beta L_{\mu}\right) \Psi^{(1)}(z, \mu, s)=\Psi^{(0)}(z, E, \mu) \\
& \mathrm{BC}: \quad \Psi^{(0)}(0, E, \mu)=10^{5} \cdot \mathrm{e}^{-200(1-\mu)^{2}} \mathrm{e}^{-50\left(E_{0}-E\right)^{2}} \quad 1 \geq \mu>0, E \in I \\
& \Psi^{(0)}(d, E, \mu)=0 \quad-1 \leq \mu<0, E \in I .
\end{aligned}
$$



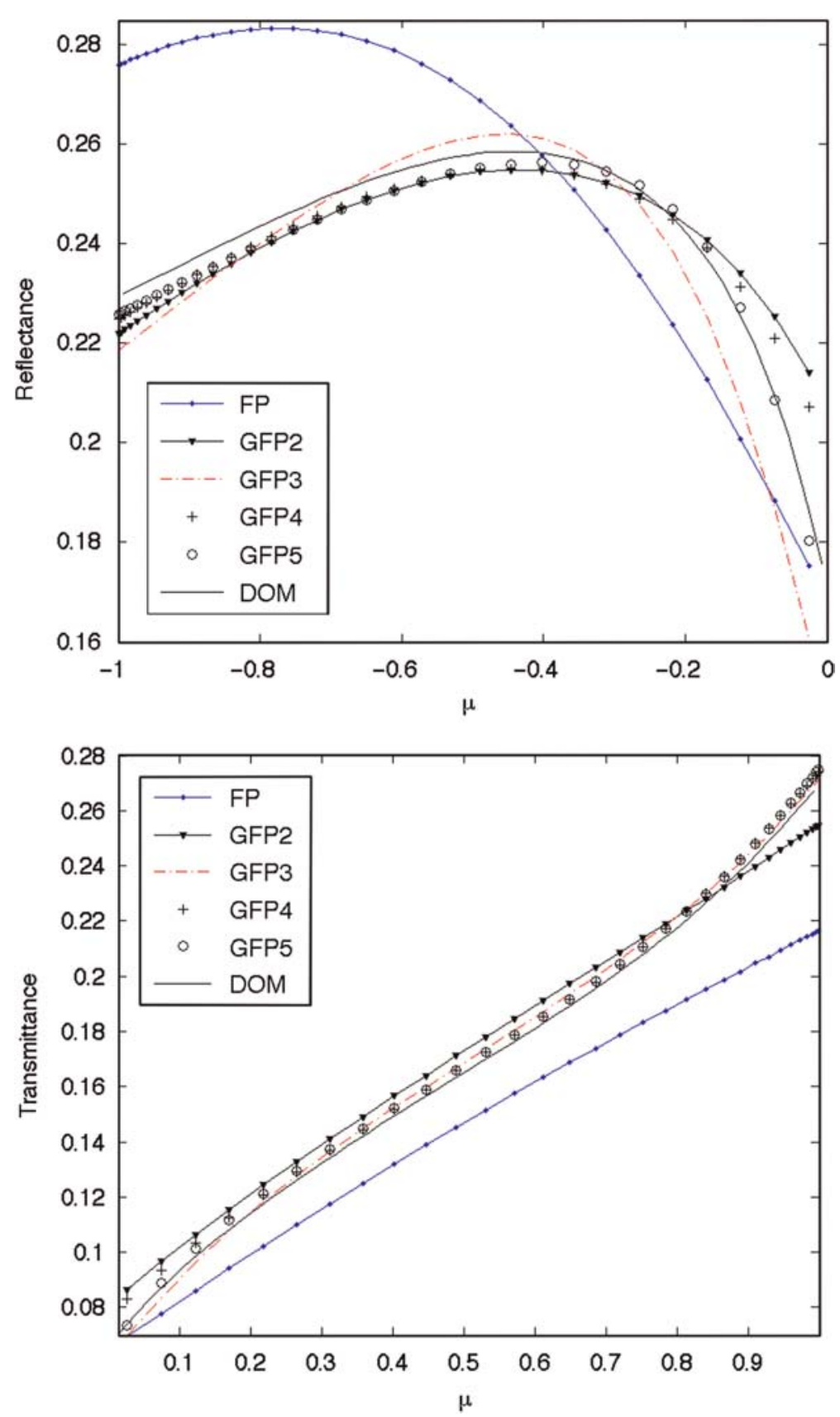

Figure 5. Double HG with $g_{1}=0.85, g_{2}=-0.34$ : GFP approximations for reflectance and transmittance of liver tissue. Transmittance is plotted in a semi-logarithmic scale. 
The initial boundary value problem in Equation (29) describes the propagation of electrons through matter with a monoenergetic pencil beam of energy $E_{0}$ irradiated orthogonally to the boundary surface of the material. This is modelled by a product of two narrow Gaussian functions around $\mu=1$ and $E=E_{0}$. After computing the solution, one can calculate the absorbed dose via

$$
D(\underline{r})=\frac{2 \pi T}{\rho(\underline{r})} \int_{0}^{\infty} \int_{-1}^{1} S\left(\underline{r}, E^{\prime}\right) \Psi^{(0)}\left(\underline{r}, \mu, E^{\prime}\right) \mathrm{d} \mu \mathrm{d} E^{\prime},
$$

where $T$ is the duration of the irradiation of the patient and $\rho$ the mass density of the irradiated tissue, so that $D(\underline{r})$ leads to SI unit $\mathrm{J} / \mathrm{kg}$ or Gy.

Several test cases were implemented for 5 and 10-MeV beams. As benchmark we used solutions of the MC code systems GEANT4 (standard physics package) [1,3] and PENELOPE [46]. However, it should be stressed that all physical models were obtained independently. The following criteria are generally employed to quantify the accuracy of a dose curve [53]: $2 \% / 2 \mathrm{~mm}$ (pointwise difference within $2 \%$ or $2 \mathrm{~mm}$ horizontal distanceto-agreement) in homogeneous and $3 \% / 3 \mathrm{~mm}$ in inhomogeneous geometries.

\subsubsection{Homogeneous geometry}

Characteristic electron dose profiles in a semi-infinite water phantom are shown in Figures 6 and 7. First they provide a high surface dose, increase to a maximum at a certain depth and drop off with a steep slope afterwards. Solutions for $\mathrm{GFP}_{4}$ and $\mathrm{GFP}_{5}$ are omitted because they overlap with $\mathrm{GFP}_{3}$ in our plot. Except for $\mathrm{GFP}_{2}$, computations were performed according to Equation (25) (32 points in $\mu, 350$ points in $z$ ). Owing to better results, we applied upwind finite difference discretizations for $\mathrm{GFP}_{2}$, equidistant in $z$ (400 points) and $\mu$ (200 points). All approximations are close to each other because GFP transport coefficients $\xi_{n}(E)$ for water do not fall off highly enough within our energy interval. All in all, the calculated results agree well with PENELOPE and GEANT4. All dose profiles for a $5-\mathrm{MeV}$ beam satisfy the $2 \% / 2 \mathrm{~mm}$ criterion. As we neglect bremsstrahlung, the difference to $\mathrm{MC}$ computations becomes bigger for $10 \mathrm{MeV}$. In fact, the largest $\mathrm{FP}$ and $\mathrm{GFP}_{2}$ distance to PENELOPE and GEANT4 becomes $3 \mathrm{~mm}$ at $z \approx 5 \mathrm{~cm}$ and hence, they do not meet the criterion.

\subsubsection{Inhomogeneous geometries}

Dose calculation is more challenging in parts of the body where materials of strongly varying densities meet. Here, large dosimetric differences between experiments and predictions exist [40]. As deviations of already 5\% in the deposited dose may result in a 20-30\% impact on complication rates [44], it is of big importance to compute the dose accurately in such transition regions.

Possible clinical applications for electron beams are, for example, irradiation of the chest wall or the vertebral column. To simulate dose curves on the central beam, we assume that $10-\mathrm{MeV}$ electrons pass three different materials: muscle $(0-1.5 \mathrm{~cm})$, bone $(1.5-3 \mathrm{~cm})$ and lung $(3-9 \mathrm{~cm})$. For all results, parameters for Morel's discretization [42] were set to 32 points in $\mu$ and 400 points in $z$. Figure 8 illustrates approximations up to order three because higher-order results overlap with the latter on that scale. The agreement with the MC dose profile is very satisfactory although bigger differences occur 


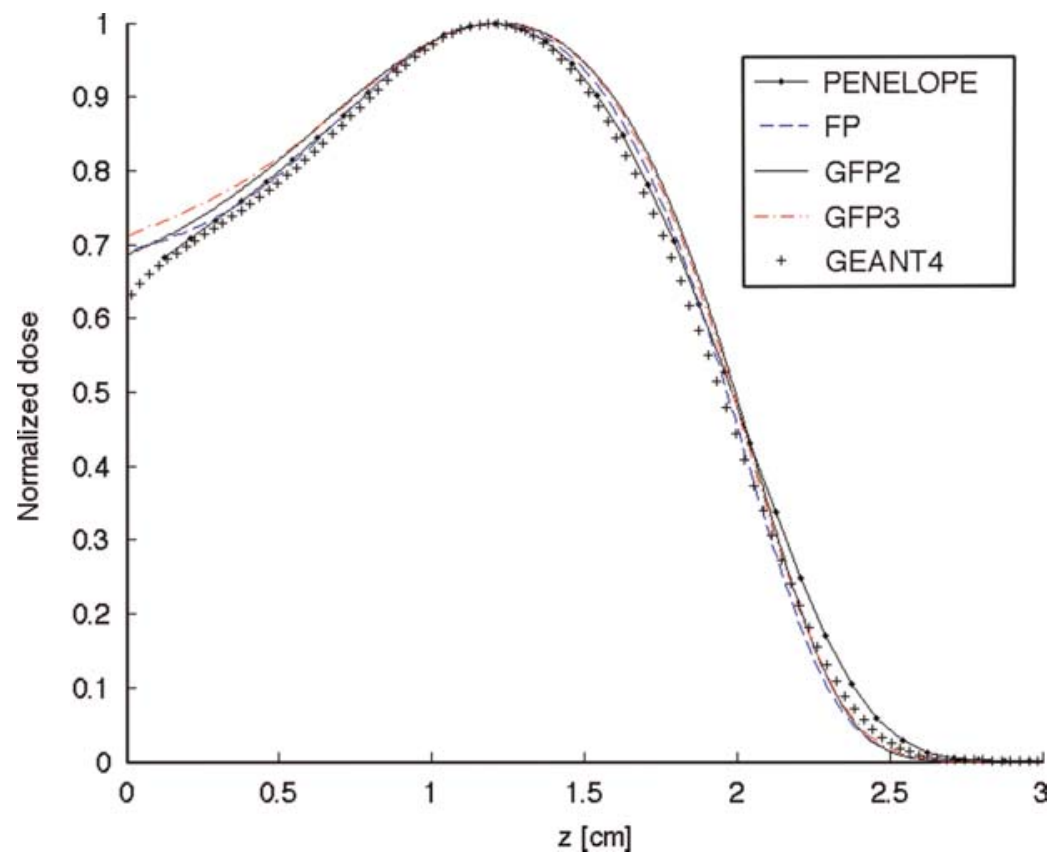

Figure 6. Normalized dose in liquid water for a $5-\mathrm{MeV}$ electron beam.

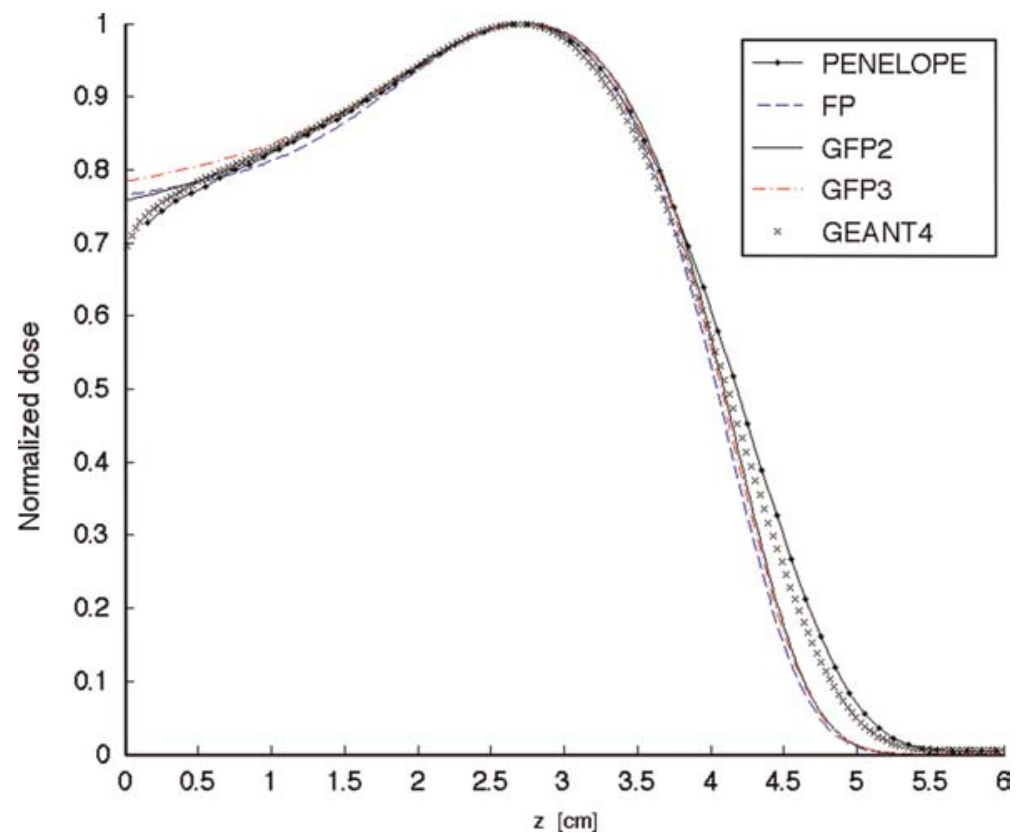

Figure 7. $10-\mathrm{MeV}$ electron beam: normalized dose in liquid water. 


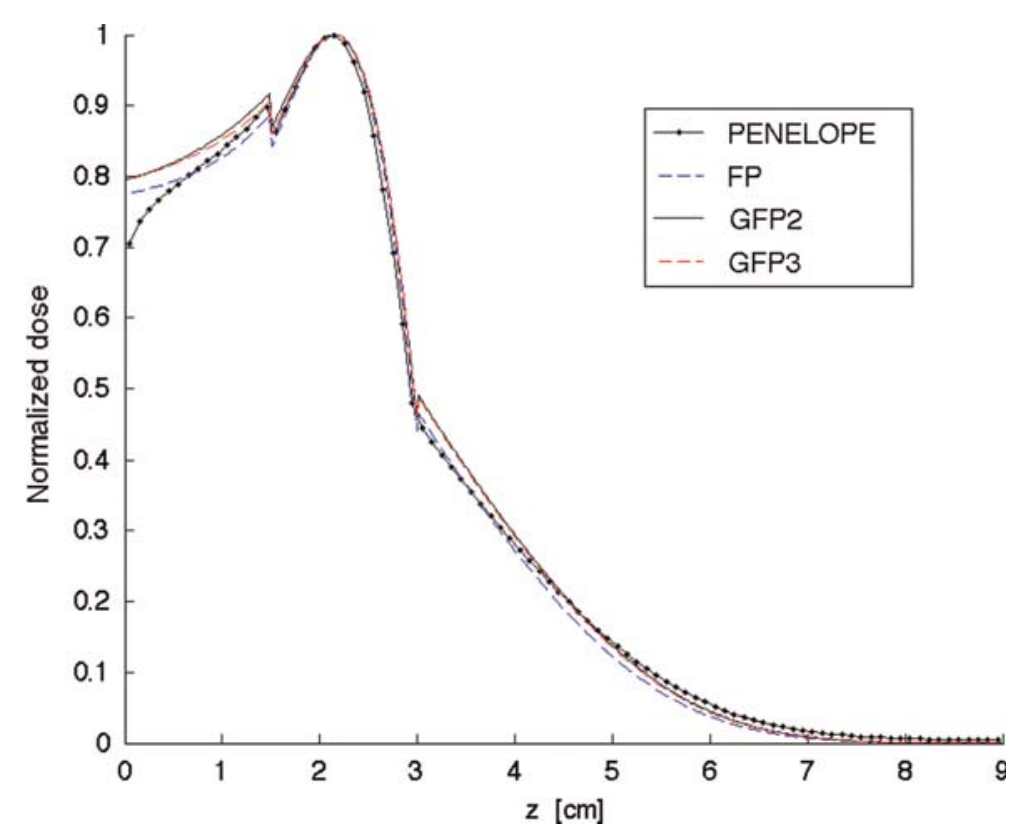

Figure 8. Normalized dose curves of $10-\mathrm{MeV}$ electrons irradiated on the back of the body.

for small penetration depths. The dose differences between PENELOPE and FP exceed the $3 \% / 3 \mathrm{~mm}$ limit only at the boundary $z=0$.

Radiotherapy gains in importance not least because surgical interventions can be avoided. Especially sensitive body areas such as the brain, coated by cerebral membranes, are of big interest. There are many voids between those membranes, which means that scattering and absorption properties change abruptly. Therefore, we consider an air cavity irradiated by a $10-\mathrm{MeV}$ electron beam first penetrating water $(0-4 \mathrm{~cm})$, then air $(4-6 \mathrm{~cm})$ and later water $(6-9 \mathrm{~cm})$ again. Similar to pure water, $\mathrm{GFP}_{2}$ calculations yield better solutions for equidistant upwind discretizations (200 points in $\mu$ and 300 points in $z$ ). Remaining curves were obtained by Morel's scheme ( $\mu$-direction: 32 points, $z$-direction: 350 points). Except for small penetration depths, all deterministic solutions are very close to each other and demonstrate good agreement with PENELOPE (Figure 9). Again FP and $\mathrm{GFP}_{2}$ results show the best approximations. Larger, but still comparably small, differences between them occur in the air region. Except for the boundary value $(z=0)$, the FP and $\mathrm{GFP}_{2}$ curves fulfill the $3 \% / 3 \mathrm{~mm}$ criterion.

\section{Conclusions}

Practical applications of GFP approximations have been at the centre of interest in this paper. Hereby, numerical examples of GFP solutions for the HG kernel in slab geometry showed more accurate approximations than FP calculations. Further test cases for reflectance and transmittance in liver tissue by means of single and double HG kernels also revealed $\mathrm{GFP}_{3}$ - and $\mathrm{GFP}_{5}$-results closest to the transport solution.

We derived an ab initio model from the ICRU database for electron transport. This model was compared to publicly available MC codes (PENELOPE and GEANT4) which in turn has been benchmarked against experiments. We extracted the stopping power, elastic and inelastic cross sections from the ICRU database and transformed them into 


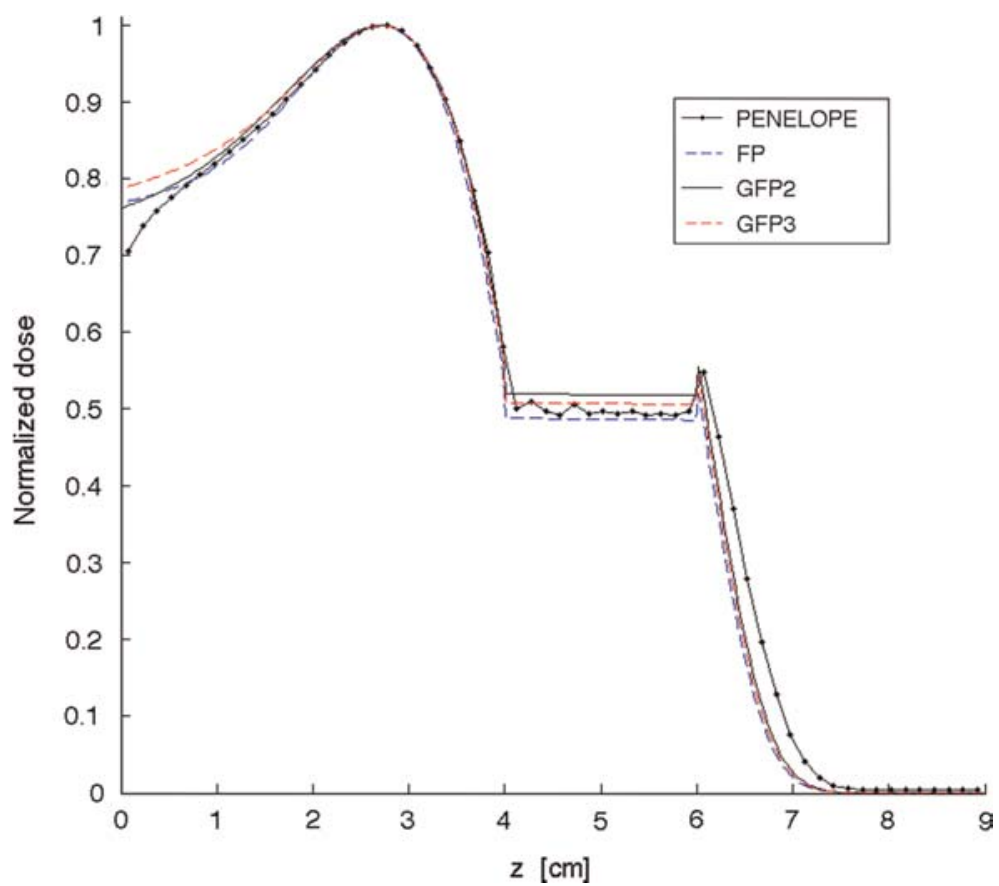

Figure 9. 10-MeV electron beam: normalized dose in liquid water with air cavity.

transport coefficients needed for GFP computations. Dose distributions for electron beams were performed without additional coupling to photons and positrons. We are aware that our physical model neglects important interactions such as energy straggling and hard radiative events with emission of photons. They are inevitable for accurate dose calculations with high-energy electrons. However, in our energy range, they are less frequent and regarded as extensions for improved models in future. And in fact, comparisons of GFP approximations with MC calculations reveal dose profiles, which are close to each other in both homogeneous and inhomogeneous geometries.

Several tasks for further examination remain:

(i) The first step towards real dose calculations from CT data is an extension to two space dimensions. As the GFP theory was derived for angular fluxes in 3D space, this should only be a challenge to the numerical and programming approach.

(ii) Owing to a rising demand for proton therapy facilities, the adaption of the GFP theory to protons is certainly an interesting subject of further study.

(iii) To improve computational results, it is necessary to include more physical phenomena. Especially for high-energy electron beams, it is inevitable to simulate the transport of bremsstrahlung quanta.

\section{Acknowledgements}

The authors would like to thank Bruno Dubroca from Université Bordeaux not only for providing his code for the DOM but also for his support and advice. We also acknowledge support from the German Research Foundation DFG under grant KL 1105/14/2 and the German Academic Exchange Service DAAD Program D/07/07534. 


\section{References}

[1] S. Agostinelli, J. Allison, K. Amako, J. Apostolakis, et al., Geant4-a simulation toolkit, Nucl. Instrum. Methods B 506 (2003), pp. 250-303.

[2] A. Ahnesjö, M. Saxner, and A. Trepp, A pencil beam model for photon dose calculation, Med. Phys. 19 (1992), pp. 263-273.

[3] J. Allison, et al., Geant4 developments and applications, IEEE Trans. Nucl. Sci. 53 (2006), pp. $270-278$.

[4] P. Andreo, Monte Carlo techniques in medical radiation physics, Phys. Med. Biol. 36 (1991), pp. 861-920.

[5] D. Balsara, Fast and accurate discrete ordinates methods for multidimensional radiative transfer. Part I, basic methods, J. Quant. Spectrosc. Radiat. Transfer 69 (2001), pp. 671-707.

[6] E. Boman, J. Tervo, and M. Vauhkonen, Modelling the transport of ionizing radiation using the finite element method, Phys. Med. Biol. 50 (2005), pp. 265-280.

[7] C. Börgers, Complexity of Monte Carlo and deterministic dose calculation methods, Phys. Med. Biol. 43 (1998), pp. 517-528.

[8] T.A. Brunner, Forms of application radiation transport. Sandia Report, sand2002-1778, Sandia National Laboratories, Albuquerque, 2002.

[9] W. Cheong, S.A. Prahl, and A.J. Welch, A review of the optical properties of biological tissues, IEEE J. Quant. Electron. 26 (1990), pp. 2166-2185.

[10] G.G.M. Coppa and P. Ravetto, Quasi-singular angular finite element methods in neutron transport problems, Trans. Theory Stat. Phys. 24 (1995), pp. 155-172.

[11] J. Cygler, et al., Clinical use of a commercial Monte Carlo treatment planning system for electron beams, Phys. Med. Biol. 50 (2005), pp. 1029-1034.

[12] R.P. Datta, et al., Computational model for coupled electron-photon transport in two dimensions, Phys. Rev. E 53 (1996), pp. 6514-6522.

[13] R. Duclous, et al., Reduced multi-scale kinetic model for the relativistic electron transport in solid targets: Effects related to secondary electrons, Laser Particle Beams 28(1) (2010), pp. $165-177$.

[14] P. Edström, A fast and stable solution method for the radiative transfer problem, SIAM Rev. 47 (2005), pp. 447-468.

[15] L. Eyges, Multiple scattering with energy loss, Phys. Rev. 74 (1948), pp. 1534-1535.

[16] E. Fermi, The ionization loss of energy in gases and in condensed materials, Phys. Rev. 57 (1940), pp. 485-493.

[17] M. Frank, H. Hensel, and A. Klar, A fast and accurate moment method for the Fokker-Planck equation and applications to electron radiotherapy, SIAM J. Appl. Math. 67 (2007), pp. 582-603.

[18] M. Frank, M. Herty, and A.N. Sandjo, Optimal treatment planning governed by kinetic equations, to appear in Math. Mod. Meth. Appl. Sci. Available at http://www.worldscinet.com/ m3as/00/forthcoming.shtml (preprint 2009).

[19] M. Frank, M. Herty, and M. Schäfer, Optimal treatment planning in radiotherapy based on Boltzmann transport calculations, Math. Mod. Meth. Appl. Sci. 18 (2008), pp. 573-592.

[20] J.C. Garth, Electron/photon transport - a key technology to radiation physics? A review, Trans. Amer. Nucl. Soc. 90 (2004), pp. 289-291.

[21] J.C. Garth, Electron/photon transport and its applications, The Monte Carlo Method: Versatility Unbounded in a Dynamic Computing World, Chattanooga, Tennessee, 17-21 April, 2005.

[22] K.A. Gifford, et al., Comparison of a finite-element multigroup discrete-ordinates code with Monte Carlo for radiotherapy calculations, Phys. Med. Biol. 51 (2006), pp. 2253-2265.

[23] P. González-Rodríguez and A.D. Kim, Light propagation in tissues with forward-peaked and large-angle scattering, Appl. Opt. 47 (2008), pp. 2599-2609.

[24] H. Hensel, R. Iza-Teran, and N. Siedow, Deterministic model for dose calculation in photon radiotherapy, Phys. Med. Biol. 51 (2006), pp. 675-693.

[25] L.G. Henyey and J.L. Greenstein, Diffuse radiation in the galaxy, Astrophys. J. 93 (1941), pp. 70-83.

[26] K.R. Hogstrom, M.D. Mills, and P.R. Almond, Electron beam dose calculations, Phys. Med. Biol. 26 (1981), pp. 445-459.

[27] H. Huizenga and P. Storchi, Numerical calculation of energy deposition by broad high-energy electron beams, Phys. Med. Biol. 34 (1989), pp. 1371-1396. 
[28] M.J. Berger, A. Jablonski, I.K. Bronic, C.J. Powell, F. Salvat, and L. Sanche, Elastic scattering of electrons and positrons, report 77, J. ICRU 7 (2007).

[29] Y. Itikawa and N. Mason, Cross sections for electron collisions with water molecules, J. Phys. Chem. Ref. Data 34(1) (2005), pp. 1-22.

[30] J. Janssen, et al., Numerical calculation of energy deposition by high-energy electron beams: III. Three-dimensional heterogeneous media, Phys. Med. Biol. 39 (1994), pp. 1351-1366.

[31] J. Janssen, et al., Numerical calculation of energy deposition by high-energy electron beams: III-B. Improvements to the 6D phase space evolution model, Phys. Med. Biol. 42 (1997), pp. $1441-1449$.

[32] D. Jette, Electron dose calculation using multiple-scattering theory. A. Gaussian multiplescattering theory, Med. Phys. 15 (1988), pp. 123-137.

[33] D. Jette and A. Bielajew, Electron dose calculation using multiple-scattering theory: Secondorder multiple-scattering theory, Med. Phys. 16 (1989), pp. 698-711.

[34] E. Korevaar, et al., Accuracy of the phase space evolution dose calculation model for clinical 25 MeV electron beams, Phys. Med. Biol. 45 (2000), pp. 2931-2945.

[35] H. Krieger, Grundlagen der Strahlungsphysik und des Strahlenschutzes, 2nd ed., Teubner Verlag, Wiesbaden, 2007.

[36] E. Larsen, et al., Electron dose calculations using the method of moments, Med. Phys. 24 (1997), pp. 111-125.

[37] J.A. LaVerne and S.M. Pimblott, Effect of elastic collisions on energy deposition by electrons in water, J. Phys. Chem. A 101 (1997), pp. 4504-4510.

[38] C.L. Leakeas and E.W. Larsen, Generalized Fokker-Planck approximations of particle transport with highly forward-peaked scattering, Nucl. Sci. Eng. 137 (2001), pp. 236-250.

[39] H.W. Lewis, Multiple scattering in an infinite medium, Phys. Rev. 78 (1950), pp. 526-529.

[40] C. Martens, et al., Underdosage of the upper-airway mucosa for small fields as used in intensity-modulated radiation therapy: A comparison between radiochromic film measurements, Monte Carlo simulations, and collapsed cone convolution calculations, Med. Phys. 29 (2002), pp. $1528-1535$.

[41] M. Morawska-Kaczyńska and H. Huizenga, Numerical calculation of energy deposition by broad high-energy electron beams: II. Multi-layered geometry, Phys. Med. Biol. 37 (1992), pp. 2103-2116.

[42] J.E. Morel, An improved Fokker-Planck angular differencing scheme, Nucl. Sci. Eng. 89 (1985), pp. 131-136.

[43] N. Papanikolau, et al., Tissue inhomogeneity corrections for megavoltage photon beams, Medical Physics Publishing, Madison, AAPM Report No. 85 (2004).

[44] G.C. Pomraning, The Fokker-Planck operator as an asymptotic limit, Math. Models Methods Appl. Sci. 2(1) (1992), pp. 21-36.

[45] B. Rossi and K. Greisen, Cosmic-ray theory, Rev. Med. Phys. 13 (1941), pp. 240-309.

[46] F. Salvat, J.M. Fernández-Varea, and J. Sempau, PENELOPE-2008: A Code System for Monte Carlo Simulation of Electron and Photon Transport, OECD, 2009.

[47] H. Schwoerer, et al., Laser-plasma acceleration of quasi-monoenergetic protons from microstructured targets, Nature 439 (2006), pp. 445-448.

[48] A.S. Shiu and K.R. Hogstrom, Pencil-beam redefinition algorithm for electron dose distributions, Med. Phys. 18 (1991), pp. 7-18.

[49] C. Siantar, et al., Description and dosimetric verification of the peregrine Monte Carlo dose calculation system for photon beams incident on a water phantom, Med. Phys. 28 (2001), pp. $1322-1337$.

[50] E. Spezi and G. Lewis, An overview of Monte Carlo treatment planning for radiotherapy, Radiat. Prot. Dos. 131 (2008), pp. 123-129.

[51] J. Tervo, et al., A finite-element model of electron transport in radiation therapy and a related inverse problem, Inverse Probl. 15 (1999), pp. 1345-1361.

[52] O.N. Vassiliev, et al., Feasibility of a multigroup deterministic solution method for $3 D$ radiotherapy dose calculations, Int. J. Radiat. Oncol. Biol. Phys. 72 (2008), pp. 220-227.

[53] J. Venselaar, H. Welleweerd, and B. Mijnheer, Tolerances for the accuracy of photon beam dose calculations of treatment planning systems, Radiother. Oncol. 60 (2001), pp. 191-201. 


\section{Appendix A. Polynomial operators}

$$
\begin{gathered}
L_{P 2}=\left(\frac{\xi_{1}}{2}+\frac{\xi_{2}}{8}\right) L+\left(\frac{\xi_{2}}{16}\right) L^{2} \\
L_{P 3}=\left(\frac{\xi_{1}}{2}+\frac{\xi_{2}}{8}+\frac{\xi_{3}}{24}\right) L+\left(\frac{\xi_{2}}{16}+\frac{\xi_{3}}{36}\right) L^{2}+\left(\frac{\xi_{3}}{288}\right) L^{3} \\
L_{P 4}=\left(\frac{\xi_{1}}{2}+\frac{\xi_{2}}{8}+\frac{\xi_{3}}{24}+\frac{\xi_{4}}{64}\right) L+\left(\frac{\xi_{2}}{16}+\frac{\xi_{3}}{36}+\frac{3 \xi_{4}}{256}\right) L^{2}+\left(\frac{\xi_{3}}{288}+\frac{5 \xi_{4}}{2304}\right) L^{3}+\left(\frac{\xi_{4}}{9216}\right) L^{4} \\
L_{P 5}=\left(\frac{\xi_{1}}{2}+\frac{\xi_{2}}{8}+\frac{\xi_{3}}{24}+\frac{\xi_{4}}{64}+\frac{\xi_{5}}{160}\right) L+\left(\frac{\xi_{2}}{16}+\frac{\xi_{3}}{36}+\frac{3 \xi_{4}}{256}+\frac{\xi_{5}}{200}\right) L^{2} \\
+\left(\frac{\xi_{3}}{288}+\frac{5 \xi_{4}}{2304}+\frac{127 \xi_{5}}{115200}\right) L^{3}+\left(\frac{\xi_{4}}{9216}+\frac{\xi_{5}}{11520}\right) L^{4}+\frac{\xi_{5}}{460800} L^{5}
\end{gathered}
$$

\section{Appendix B. Derivation of GFP operators}

\section{$\mathrm{GFP}_{2}$}

If $\lambda_{n}^{\mathrm{B}}$ denotes one eigenvalue of $L_{\mathrm{B}}$ for $n \geq 0$ and $\alpha, \beta$ are two positive constants, the GFP operator defined by

$$
L_{P 2}+\mathcal{O}\left(\varepsilon^{2}\right)=L_{\mathrm{GFP}_{2}}:=\alpha L(I-\beta L)^{-1} ;=\alpha L+\alpha \beta L^{2}+\mathcal{O}\left(\alpha \beta^{2}\right),
$$

will have to satisfy three properties to substitute $L_{P 2}$ in the favoured way:

(1) Eigenvalue preservation:

$$
-\frac{\alpha n(n+1)}{1+\beta n(n+1)}=\lambda_{n}^{\mathrm{GFP}_{2}} \stackrel{!}{=} \lambda_{n}^{\mathrm{B}}=-\sigma_{a n} \quad \text { for } n=1,2
$$

Multiplying the above equation by $(1+\beta n(n+1)) \neq 0$ and dividing by $n(n+1)$, we conclude

$$
\left(\alpha-\beta \sigma_{a n}\right)=\frac{\sigma_{a n}}{n(n+1)} \quad n=1,2 \Leftrightarrow\left[\begin{array}{c}
1-\sigma_{a 1} \\
1-\sigma_{a 2}
\end{array}\right] \cdot\left[\begin{array}{l}
\alpha \\
\beta
\end{array}\right]=\left[\begin{array}{l}
\sigma_{a 1} / 2 \\
\sigma_{a 2} / 6
\end{array}\right]
$$

(2) Order: $\mathcal{O}\left(\alpha \beta^{2}\right) \stackrel{!}{=} \mathcal{O}\left(\varepsilon^{2}\right)$

(3) Equivalence:

$$
\alpha L+\alpha \beta L^{2} \stackrel{!}{=} L_{P 2}+\mathcal{O}\left(\varepsilon^{2}\right)=\left(\frac{\xi_{1}}{2}+\frac{\xi_{2}}{8}\right) L+\frac{\xi_{2}}{16} L^{2}+\mathcal{O}\left(\varepsilon^{2}\right)
$$


where $\sigma_{a n}$ is a quantity which can be expressed in terms of $\xi_{n}$ :

$$
\begin{gathered}
\sigma_{a 0}=0, \\
\sigma_{a 1}=\xi_{1}, \\
\sigma_{a 2}=3 \xi_{1}-\frac{3}{2} \xi_{2}, \\
\sigma_{a 3}=6 \xi_{1}-\frac{15}{2} \xi_{2}+\frac{5}{2} \xi_{3}, \\
\sigma_{a 4}=10 \xi_{1}-\frac{45}{2} \xi_{2}+\frac{35}{2} \xi_{3}-\frac{35}{8} \xi_{4}, \\
\sigma_{a 5}=15 \xi_{1}-\frac{105}{2} \xi_{2}+70 \xi_{3}-\frac{315}{8} \xi_{4}+\frac{63}{8} \xi_{5} .
\end{gathered}
$$

In the following, item (1) is first transformed to a system of linear equations and thereafter solved for the desired GFP coefficients (here $\alpha$ and $\beta$ ). However, the final equations to be solved are non-linear for GFP operators of order $n \geq 3$. In this case of order $n=2$, Equations (B3) and (B4) yield

$$
\alpha=\frac{\xi_{1}}{2}+\frac{\xi_{2}}{8} \quad \text { and } \quad \beta=\frac{\xi_{2}}{8 \xi_{1}} \text {. }
$$

Going on with item (2), it is to be checked

$$
\alpha \beta^{2}=\left(\frac{\xi_{1}}{2}+\frac{\xi_{2}}{8}\right)\left(\frac{\xi_{2}}{8 \xi_{1}}\right)^{2}=\underbrace{\left(\frac{\xi_{1}}{2}+\frac{\xi_{2}}{8}\right)}_{\in \mathcal{O}(1)} \underbrace{\left(\frac{\xi_{2}^{2}}{64 \xi_{1}^{2}}\right)}_{\in \mathcal{O}\left(\varepsilon^{2}\right)} \in \mathcal{O}\left(\varepsilon^{2}\right) .
$$

As equivalence condition follows straight forward, it has been shown that the operator $L_{\mathrm{GFP}_{2}}$ is an $\mathcal{O}\left(\varepsilon^{2}\right)$ approximation to $L_{\mathrm{B}}$ whose first three eigenvalues agree. Now it is quite intuitive to apply a similar procedure to higher-order operators. We determined explicit solutions for $\mathrm{GFP}_{2}-$ $\mathrm{GFP}_{5}$ coefficients and performed verifications for items (1)-(3). According to $\mathrm{GFP}_{3}$ all items were checked without computer support. The asymptotic behaviour of GFP operators of order four and five was, however, checked by means of a symbolic toolbox. To keep our following description short, we confine ourselves to final results.

\section{$\mathrm{GFP}_{3}$}

$$
\begin{aligned}
L_{P 3}+\mathcal{O}\left(\varepsilon^{3}\right) & =L_{\mathrm{GFP}_{3}}:=\alpha_{1} L\left(I-\beta_{1} L\right)^{-1}+\alpha_{2} L \\
& =\left(\alpha_{1}+\alpha_{2}\right) L+\alpha_{1} \beta_{1} L^{2}+\alpha_{1} \beta_{1}^{2} L^{3}+\mathcal{O}\left(\alpha_{1} \beta_{1}^{3}\right)
\end{aligned}
$$


(1) Eigenvalue preservation:

$$
\begin{gathered}
\left(\alpha_{1}+\alpha_{2}\right)-\sigma_{a n} \beta_{1}+n(n+1) \beta_{1} \alpha_{2}=\frac{\sigma_{a n}}{n(n+1)} \quad n=1,2,3 \\
\Rightarrow \alpha_{1}=\frac{\xi_{2}\left(27 \xi_{2}^{2}+5 \xi_{3}^{2}-24 \xi_{2} \xi_{3}\right)}{8 \xi_{3}\left(3 \xi_{2}-2 \xi_{3}\right)} \\
\beta_{1}=\frac{\xi_{3}}{6\left(3 \xi_{2}-2 \xi_{3}\right)} \\
\alpha_{2}=\frac{\xi_{1}}{2}-\frac{9 \xi_{2}^{2}}{8 \xi_{3}}+\frac{3 \xi_{2}}{8} .
\end{gathered}
$$

(2) Equivalence: To guarantee that $L_{\mathrm{GFP}_{3}}=L_{P 3}+\mathcal{O}\left(\varepsilon^{3}\right)$, all coefficients of $L^{i}$ in Equations (B8) and (A2) must coincide. Let $\alpha_{1}, \beta_{1}, \alpha_{2}$ be the defining positive coefficients of $L_{\mathrm{GFP}_{3}}$ as stated in Equations (B10)-(B12), then one can show that they satisfy

$$
\begin{gathered}
\text { I. } \alpha_{1}+\alpha_{2}=\frac{\xi_{1}}{2}+\frac{\xi_{2}}{8}+\frac{\xi_{3}}{24}+\mathcal{O}\left(\varepsilon^{3}\right) \\
\text { II. } \quad \alpha_{1} \beta_{1}=\frac{\xi_{2}}{16}+\frac{\xi_{3}}{36}+\mathcal{O}\left(\varepsilon^{3}\right) \\
\text { III. } \quad \alpha_{1} \beta_{1}^{2}=\frac{\xi_{3}}{288}+\mathcal{O}\left(\varepsilon^{3}\right) .
\end{gathered}
$$

\section{$\mathrm{GFP}_{4}$}

$$
\begin{aligned}
L_{P 4}+\mathcal{O}\left(\varepsilon^{4}\right)= & L_{\mathrm{GFP}_{4}}:=\alpha_{1} L\left(I-\beta_{1} L\right)^{-1}+\alpha_{2} L\left(I-\beta_{2} L\right)^{-1} \\
= & \left(\alpha_{1}+\alpha_{2}\right) L+\left(\alpha_{1} \beta_{1}+\alpha_{2} \beta_{2}\right) L^{2}+\left(\alpha_{1} \beta_{1}^{2}+\alpha_{2} \beta_{2}^{2}\right) L^{3}+\left(\alpha_{1} \beta_{1}^{3}+\alpha_{2} \beta_{2}^{3}\right) L^{4} \\
& +\mathcal{O}\left(\alpha_{1} \beta_{1}^{4}\right)+\mathcal{O}\left(\alpha_{2} \beta_{2}^{4}\right)
\end{aligned}
$$

(1) Eigenvalue preservation:

$$
\begin{aligned}
& \left(\alpha_{1}+\alpha_{2}\right)-\sigma_{a n}\left(\beta_{1}+\beta_{2}\right)-\sigma_{a n} n(n+1) \beta_{1} \beta_{2}+n(n+1)\left(\alpha_{1} \beta_{2}+\alpha_{2} \beta_{1}\right) \\
& =\frac{\sigma_{a n}}{n(n+1)} \quad n=1,2,3,4
\end{aligned}
$$

(2) Equivalence:

$$
\begin{gathered}
\text { I. } \quad \alpha_{1}+\alpha_{2}=\frac{\xi_{1}}{2}+\frac{\xi_{2}}{8}+\frac{\xi_{3}}{24}+\frac{\xi_{4}}{64}+\mathcal{O}\left(\varepsilon^{4}\right) \\
\text { II. } \quad \alpha_{1} \beta_{1}+\alpha_{2} \beta_{2}=\frac{\xi_{2}}{16}+\frac{\xi_{3}}{36}+\frac{3 \xi_{4}}{256}+\mathcal{O}\left(\varepsilon^{4}\right) \\
\text { III. } \quad \alpha_{1} \beta_{1}^{2}+\alpha_{2} \beta_{2}^{2}=\frac{\xi_{3}}{288}+\frac{5 \xi_{4}}{2304}+\mathcal{O}\left(\varepsilon^{4}\right) \\
\text { IV. } \quad \alpha_{1} \beta_{1}^{3}+\alpha_{2} \beta_{2}^{3}=\frac{\xi_{4}}{9216}+\mathcal{O}\left(\varepsilon^{4}\right)
\end{gathered}
$$


$\mathrm{GFP}_{5}$

$$
\begin{aligned}
L_{P 5}+\mathcal{O}\left(\varepsilon^{5}\right) & =L_{\mathrm{GFP}_{5}}:=\alpha_{1} L\left(I-\beta_{1} L\right)^{-1}+\alpha_{2} L\left(I-\beta_{2} L\right)^{-1}+\alpha_{3} L \\
& =\left(\alpha_{1}+\alpha_{2}+\alpha_{3}\right) L+\left(\alpha_{1} \beta_{1}+\alpha_{2} \beta_{2}\right) L^{2}+\left(\alpha_{1} \beta_{1}^{2}+\alpha_{2} \beta_{2}^{2}\right) L^{3}+\left(\alpha_{1} \beta_{1}^{3}+\alpha_{2} \beta_{2}^{3}\right) L^{4} \\
& +\left(\alpha_{1} \beta_{1}^{4}+\alpha_{2} \beta_{2}^{4}\right) L^{5}+\mathcal{O}\left(\alpha_{1} \beta_{1}^{5}\right)+\mathcal{O}\left(\alpha_{2} \beta_{2}^{5}\right)
\end{aligned}
$$

(1) Eigenvalue preservation:

$$
\begin{aligned}
& \left(\alpha_{1}+\alpha_{2}+\alpha_{3}\right)-\sigma_{a n}\left(\beta_{1}+\beta_{2}\right)-\sigma_{a n} n(n+1) \beta_{1} \beta_{2} \\
& +n(n+1)\left[\beta_{2}\left(\alpha_{1}+\alpha_{3}\right)+\beta_{1}\left(\alpha_{2}+\alpha_{3}\right)\right]+\alpha_{3} \beta_{1} \beta_{2}[n(n+1)]^{2}=\frac{\sigma_{a n}}{n(n+1)} \\
& n=1,2,3,4,5
\end{aligned}
$$

(2) Equivalence:

$$
\begin{gathered}
\text { I. } \alpha_{1}+\alpha_{2}+\alpha_{3}=\frac{\xi_{1}}{2}+\frac{\xi_{2}}{8}+\frac{\xi_{3}}{24}+\frac{\xi_{4}}{64}+\frac{\xi_{5}}{160}+\mathcal{O}\left(\varepsilon^{5}\right) \\
\text { II. } \quad \alpha_{1} \beta_{1}+\alpha_{2} \beta_{2}=\frac{\xi_{2}}{16}+\frac{\xi_{3}}{36}+\frac{3 \xi_{4}}{256}+\frac{\xi_{5}}{200}+\mathcal{O}\left(\varepsilon^{5}\right) \\
\text { III. } \quad \alpha_{1} \beta_{1}^{2}+\alpha_{2} \beta_{2}^{2}=\frac{\xi_{3}}{288}+\frac{5 \xi_{4}}{2304}+\frac{127 \xi_{5}}{115200}+\mathcal{O}\left(\varepsilon^{5}\right) \\
\text { IV. } \alpha_{1} \beta_{1}^{3}+\alpha_{2} \beta_{2}^{3}=\frac{\xi_{4}}{9216}+\frac{\xi_{5}}{11520}+\mathcal{O}\left(\varepsilon^{5}\right) \\
\text { V. } \alpha_{1} \beta_{1}^{4}+\alpha_{2} \beta_{2}^{4}=\frac{\xi_{5}}{460800}+\mathcal{O}\left(\varepsilon^{5}\right)
\end{gathered}
$$

All linear and nonlinear equations stated above lead to explicit solutions for $\alpha_{i}$ and $\beta_{i}$. Equations posed for $\mathrm{GFP}_{2}$ and $\mathrm{GFP}_{3}$ actually deliver uniquely determined constants, whereas for higher GFP operators there is no guarantee for unique or even real-valued $\alpha_{i}$ and $\beta_{i}$. Nevertheless, it is important to emphasize that the resulting values of $\alpha_{i}$ and $\beta_{i}$ must be positive. Otherwise, eigenvalues $\lambda_{n}^{\mathrm{GFP}_{k}}$ of a $\mathrm{GFP}_{k}$ operator could become negative. 


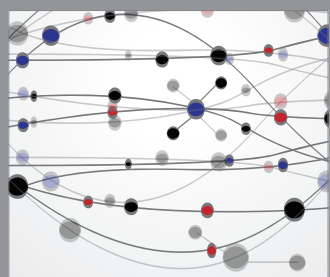

The Scientific World Journal
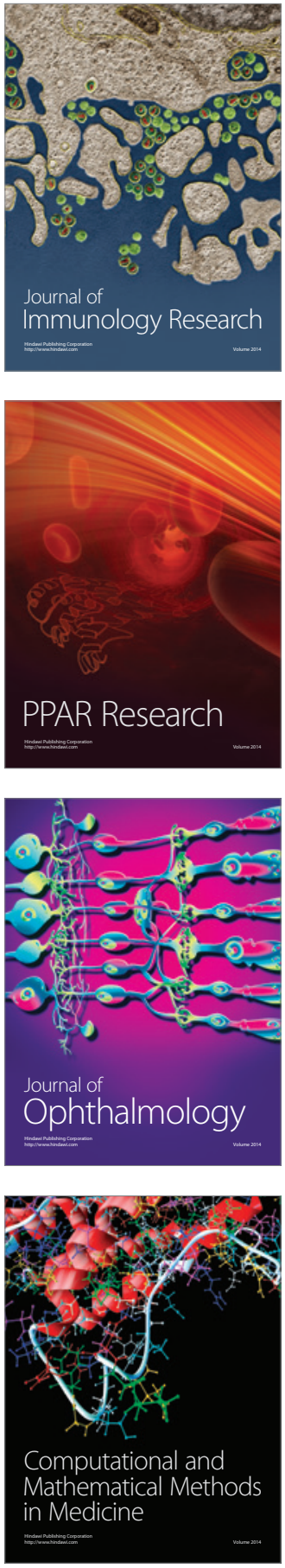

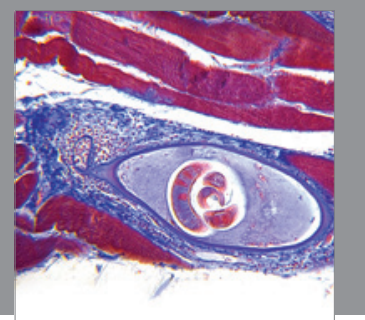

Gastroenterology

Research and Practice
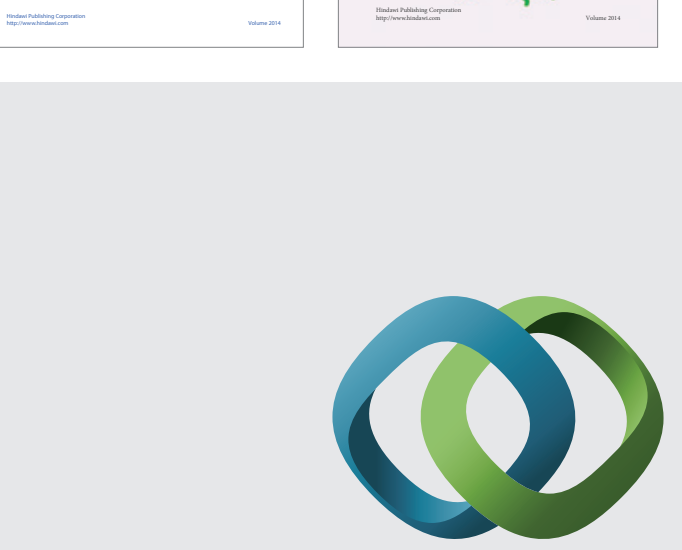

\section{Hindawi}

Submit your manuscripts at

http://www.hindawi.com
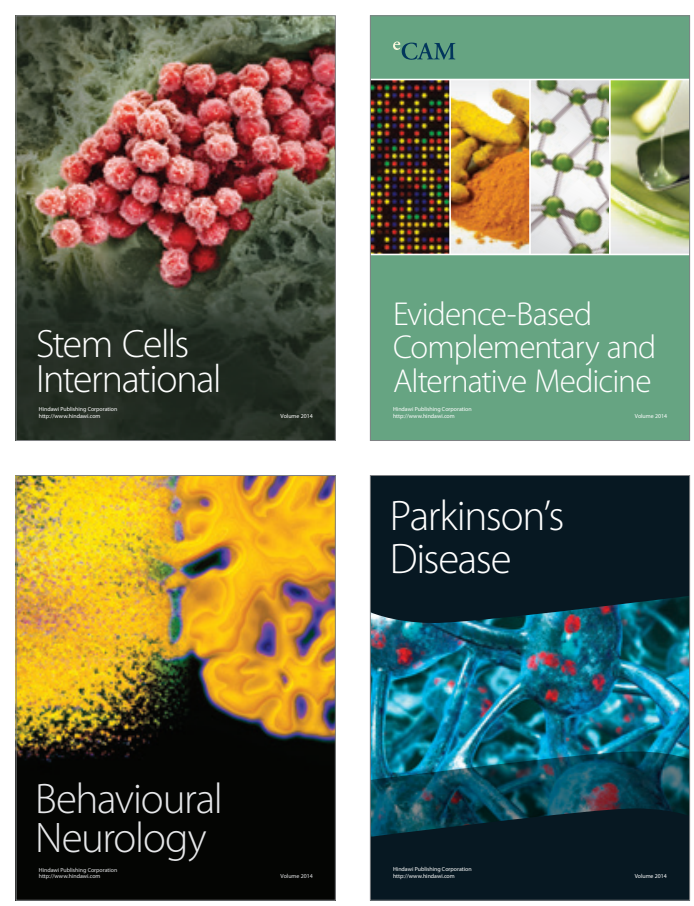

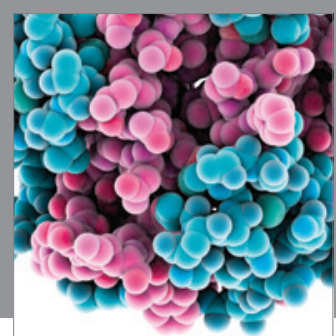

Journal of
Diabetes Research

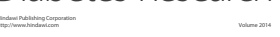

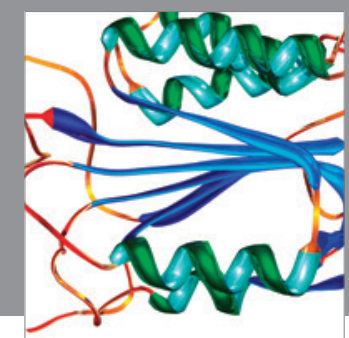

Disease Markers
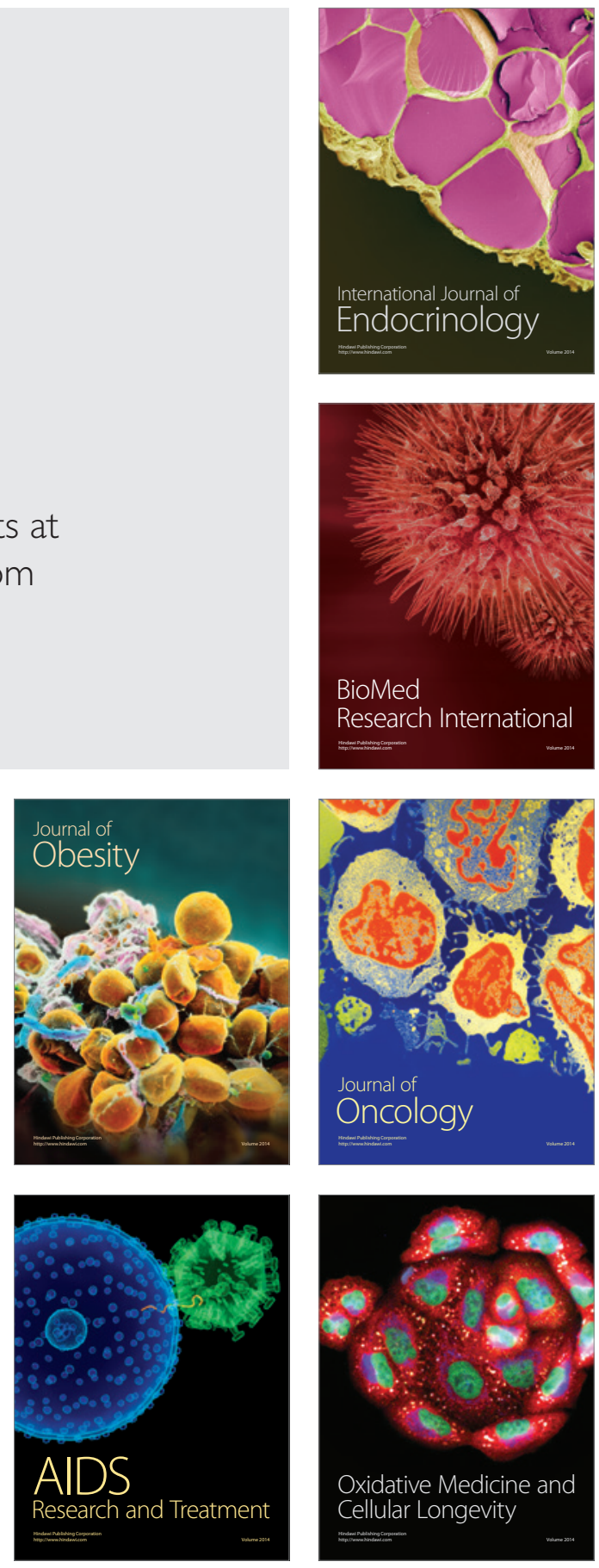